\title{
Ab initio calculations for Gamow-Teller strengths in sd shell
}

\author{
Archana Saxena ${ }^{1 *}$, Praveen C. Srivastava ${ }^{1 \dagger}$ and Toshio Suzuki ${ }^{2,3 \ddagger}$ \\ ${ }^{1}$ Department of Physics, Indian Institute of Technology Roorkee, Roorkee 247 667, India \\ ${ }^{2}$ Department of Physics, College of Humanities and Science, \\ Nihon Univerity, Sakurajosui 3, Setagaya-ku, Tokyo 156-8550, Japan and \\ ${ }^{3}$ National Astronomical Observatory of Japan, Osawa 2, Mitaka, Tokyo 181-8588, Japan
}

(Dated: January 16, 2018)

\begin{abstract}
In the present work we perform a systematic shell model study of Gamow-Teller transition strength distributions in $s d$ shell nuclei using $a b$ initio effective interactions. The $a b$ initio effective interactions are based on in-medium similarity renormalization group (IM-SRG) and coupled-cluster effective interaction (CCEI) approaches. The aim of the present work is to test the predictive power of $a b$ initio effective interactions by using the available experimental data of Gamow-Teller strength distributions in $s d$ shell nuclei. We perform calculations for ${ }^{20} \mathrm{Ne} \rightarrow{ }^{20} \mathrm{~F},{ }^{23} \mathrm{Na} \rightarrow{ }^{23} \mathrm{Mg},{ }^{23} \mathrm{Na} \rightarrow$ ${ }^{23} \mathrm{Ne},{ }^{24} \mathrm{Mg} \rightarrow{ }^{24} \mathrm{Na},{ }^{24} \mathrm{Mg} \rightarrow{ }^{24} \mathrm{Al},{ }^{25} \mathrm{Mg} \rightarrow{ }^{25} \mathrm{Al},{ }^{26} \mathrm{Mg} \rightarrow{ }^{26} \mathrm{Na},{ }^{26} \mathrm{Mg} \rightarrow{ }^{26} \mathrm{Al},{ }^{26} \mathrm{Si} \rightarrow{ }^{26} \mathrm{Al},{ }^{27} \mathrm{Al} \rightarrow$ ${ }^{27} \mathrm{Si},{ }^{28} \mathrm{Si} \rightarrow{ }^{28} \mathrm{P},{ }^{31} \mathrm{P} \rightarrow{ }^{31} \mathrm{Si}$, and ${ }^{32} \mathrm{~S} \rightarrow{ }^{32} \mathrm{P}$ transitions. For comparison we also show the results obtained by using the phenomenological USDB Hamiltonian. The phenomenological USDB results of the Gamow-Teller $\left(\mathrm{GT}_{+} / \mathrm{GT}_{-}\right)$strength distributions show reasonable agreements with the experimental data in comparison to the ab initio interactions. We also calculate the electron capture reaction rates for ${ }^{23} \mathrm{Na}\left(\mathrm{e}^{-}, \nu\right)^{23} \mathrm{Ne}$ and ${ }^{25} \mathrm{Mg}\left(\mathrm{e}^{-}, \nu\right){ }^{25} \mathrm{Na}$ using ab initio and USDB interactions.

PACS numbers: 21.60.Cs, 21.60.Ev, 27.50.+e, 23.40.-s
\end{abstract}

\section{INTRODUCTION}

The Gamow-Teller (GT) transitions are one of the important tools to explore the structure of atomic nuclei $[1-6]$. It has many applications such as $\beta$-decay in stellar evolution and electron capture [7-10]; double electron capture for heating of stars [11] and neutrino nucleosynthesis [12-15]. There are two types of GT transitions, the $\mathrm{GT}_{+}$transition where a proton changes into a neutron, and the $\mathrm{GT}_{-}$transition where a neutron changes into a proton. The experimental $B(G T)$ strengths can be obtained from $\beta$-decay studies, but the excitation energies are limited by the decay $Q$-values in this approach. On the other hand, with the charge exchange (CE) reactions, such as $(p, n),(n, p),\left(d,{ }^{2} \mathrm{He}\right)$ or $\left({ }^{3} \mathrm{He}, t\right)$, it is possible to access GT transitions at higher energies without the $Q$-value limitation. The experimental measurements at scattering angles around $0^{\circ}$ and incident energies above $100 \mathrm{MeV} /$ nucleon provide valuable information on the GT transitions.

There are various experimental probes for the measurement of the GT strengths in $s d$ shell nuclei in $A=20-32$ mass region. For the $\mathrm{GT}_{+}$transition, the $(n, p)$ and $\left(d,{ }^{2} \mathrm{He}\right)$ reactions are mainly used to obtain the strength distribution. The $\left(t,{ }^{3} \mathrm{He}\right)$ reaction is also an alternative tool. The GT transition strengths extracted from $\beta$-decay and $\mathrm{CE}$ reactions provide also important tests for ab initio calculations.

Modern ab initio approaches, like the IM-SRG [16], the coupled cluster theory [17] and the self-consistent Green's

\footnotetext{
*archanasaxena777@gmail.com

${ }^{\dagger}$ pcsrifph@iitr.ac.in

${ }^{\ddagger}$ suzuki@chs.nihon-u.ac.jp
}

function method [18], have been established and provide accurate description of nuclear structure properties. The ab initio approaches are more fundamental, although in many cases empirical interactions still are used as benchmarks. The $a b$ initio calculations can be used not only for spherical nuclei, but also to predict the ground and excited state energies and deformations for doubly openshell nuclei [19]. The yrast deformed states in ${ }^{20} \mathrm{Ne}$ and ${ }^{24} \mathrm{Mg}$ have been recently reported in Ref. [19].

The aim of the present study is to calculate the GT strengths for $s d$ shell nuclei using ab initio approaches. In Table I, we give a list of $s d$ shell nuclei and corresponding types of reactions, for which we perform the shell model calculations. Thus, the present work will add more information to earlier $B(G T)$ results for $s d$ shell nuclei obtained by using phenomenological interactions. Previously, the $B(G T)$ strengths for $A=10-13$ ( $p$ shell) nuclei, using the no-core shell model with twonucleon $(N N)$ and three-nucleon $(3 N)$ interactions derived from chiral effective field theory, were reported in Ref. [20]. Results for the GT strength in the ${ }^{7} \mathrm{Be}\left(3 / 2_{g . s}^{-}\right)$ $\rightarrow{ }^{7} \operatorname{Li}\left(3 / 2_{g . s}^{-}, 1 / 2_{1}^{-}\right)$transition, obtained by using the nocore shell model with the Argonne $\mathrm{V}^{\prime} N N$ potential and the Tucson-Melbourne $\mathrm{TM}^{\prime}(99) 3 N$ potential, were reported in Ref. [21].

In the next section, we discuss details on the $a b$ initio effective interactions, along with the phenomenological USDB interaction and the GT operator. We also discuss the Ikeda sum rule to check the predictive power of $a b$ initio interactions. In section III, comparison between the experimental and calculated GT strengths for 13 sets of transitions are reported. In section IV, we discuss the electron capture rates. Finally, a summary of the present work is given in Section V. 
TABLE I: List of the GT transitions studied in this work. The data types available and the types of theoretical calculations used are given. In the last column the references for the data sets, which are used for comparison with the theoretical calculations, are given.

\begin{tabular}{|c|c|c|c|c|c|c|c|c|c|c|c|c|}
\hline S.No. & Initial & Final & $\beta$-decay & $(n, p)$ & $\left(d,{ }^{2} \mathrm{He}\right)$ & $\left(t,{ }^{3} \mathrm{He}\right)$ & $\left({ }^{3} \mathrm{He}, t\right)$ & $(p, n)$ & CCEI & IM-SRG & USDB & Ref. \\
\hline 1. & ${ }^{20} \mathrm{Ne}\left(0^{+}\right)$ & ${ }^{20} \mathrm{~F}\left(1^{+}\right)$ & & $\sqrt{ }$ & & & & & $\sqrt{ }$ & $\sqrt{ }$ & $\sqrt{ }$ & [29] \\
\hline 2. & ${ }^{23} \mathrm{Na}\left(\frac{3}{2}^{+}\right)$ & ${ }^{23} \mathrm{Mg}\left(\frac{1}{2}^{+}, \frac{3}{2}^{+}, \frac{5}{2}^{+}\right)$ & & & & & $\sqrt{ }$ & & $\sqrt{ }$ & $\sqrt{ }$ & $\sqrt{ }$ & {$[30]$} \\
\hline 3. & ${ }^{23} \mathrm{Na}\left(\frac{3}{2}^{+}\right)$ & ${ }^{23} \mathrm{Ne}\left(\frac{1}{2}^{+}, \frac{3}{2}^{+}, \frac{5}{2}^{+}\right)$ & & $\sqrt{ }$ & & & & & $\sqrt{ }$ & $\sqrt{ }$ & $\sqrt{ }$ & {$[31]$} \\
\hline 4. & ${ }^{24} \mathrm{Mg}\left(0^{+}\right)$ & ${ }^{24} \mathrm{Na}\left(1^{+}\right)$ & & & $\sqrt{ }$ & $\sqrt{ }$ & & & $\sqrt{ }$ & $\sqrt{ }$ & $\sqrt{ }$ & {$[32,33]$} \\
\hline 5. & ${ }^{24} \mathrm{Mg}\left(0^{+}\right)$ & ${ }^{24} \mathrm{Al}\left(1^{+}\right)$ & & & & & $\sqrt{ }$ & $\sqrt{ }$ & $\sqrt{ }$ & $\sqrt{ }$ & $\sqrt{ }$ & {$[34,35]$} \\
\hline 6. & ${ }^{25} \mathrm{Mg}\left(\frac{5}{2}^{+}\right)$ & ${ }^{25} \mathrm{Al}\left(\frac{3}{2}^{+}, \frac{5}{2}^{+}, \frac{7}{2}^{+}\right)$ & & & & & $\sqrt{ }$ & & $\sqrt{ }$ & $\sqrt{ }$ & $\sqrt{ }$ & {$[36]$} \\
\hline 7. & ${ }^{26} \mathrm{Mg}\left(0^{+}\right)$ & ${ }^{26} \mathrm{Na}\left(1^{+}\right)$ & & & $\sqrt{ }$ & $\sqrt{ }$ & & & $\sqrt{ }$ & $\sqrt{ }$ & $\sqrt{ }$ & {$[37,38]$} \\
\hline 8. & ${ }^{26} \mathrm{Mg}\left(0^{+}\right)$ & ${ }^{26} \mathrm{Al}\left(1^{+}\right)$ & & & & & $\sqrt{ }$ & $\sqrt{ }$ & $\sqrt{ }$ & $\sqrt{ }$ & $\sqrt{ }$ & {$[37,39]$} \\
\hline 9. & ${ }^{26} \mathrm{Si}\left(0^{+}\right)$ & ${ }^{26} \mathrm{Al}\left(1^{+}\right)$ & $\sqrt{ }$ & & & & & & $\sqrt{ }$ & $\sqrt{ }$ & $\sqrt{ }$ & {$[40]$} \\
\hline 10. & ${ }^{27} \mathrm{Al}\left(\frac{5}{2}^{+}\right)$ & ${ }^{27} \mathrm{Si}\left(\frac{3}{2}^{+}, \frac{5}{2}^{+}, \frac{7}{2}^{+}\right)$ & & & & & $\sqrt{ }$ & & $\sqrt{ }$ & $\sqrt{ }$ & $\sqrt{ }$ & [41] \\
\hline 11. & ${ }^{28} \mathrm{Si}\left(0^{+}\right)$ & ${ }^{28} \mathrm{P}\left(1^{+}\right)$ & & & & & $\sqrt{ }$ & $\sqrt{ }$ & $\sqrt{ }$ & $\sqrt{ }$ & $\sqrt{ }$ & {$[34,42]$} \\
\hline 12 . & ${ }^{31} \mathrm{P}\left(\frac{1}{2}^{+}\right)$ & ${ }^{31} \mathrm{Si}\left(\frac{1}{2}^{+}, \frac{3}{2}^{+}\right)$ & & $\sqrt{ }$ & & & & & $\sqrt{ }$ & $\sqrt{ }$ & $\sqrt{ }$ & {$[43]$} \\
\hline 13. & ${ }^{32} \mathrm{~S}\left(0^{+}\right)$ & ${ }^{32} \mathrm{P}\left(1^{+}\right)$ & & & $\sqrt{ }$ & & & & $\sqrt{ }$ & $\sqrt{ }$ & $\sqrt{ }$ & [44] \\
\hline
\end{tabular}

\section{DETAILS ON $a b$ initio CALCULATIONS}

In order to describe the measured GT strength distribution in $s d$ shell nuclei, we have performed shell model calculations with two modern ab initio approaches: The in-medium similarity renormalization group (IM-SRG) [19] and the coupled cluster effective interaction (CCEI) [22-24]. For comparison, we have also performed calculations with the phenomenological USDB effective interaction [25]. For the diagonalization of matrices we used the shell-model code NuShellX [26].

Stroberg et al. [19] derived a mass-dependent Hamiltonian for $s d$ shell nuclei by using the IM-SRG [27] based on chiral two- and three-nucleon interactions. In this method, an initial Hamiltonian $H$, which is normal ordered with respect to a finite-density reference state $|\Phi\rangle$ (e.g., the Hartree-Fock ground state) is given as:

$$
\begin{array}{r}
H=E_{0}+\sum_{i j} f_{i j}\left\{a_{i}^{\dagger} a_{j}\right\}+\frac{1}{2 !^{2}} \sum_{i j k l} \Gamma_{i j k l}\left\{a_{i}^{\dagger} a_{j}^{\dagger} a_{l} a_{k}\right\} \\
+\frac{1}{3 !^{2}} \sum_{i j k l m n} W_{i j k l m n}\left\{a_{i}^{\dagger} a_{j}^{\dagger} a_{k}^{\dagger} a_{n} a_{m} a_{l}\right\}
\end{array}
$$

where, $E_{0}, f_{i j}, \Gamma_{i j k l}$ and $W_{i j k l m n}$ are the normal ordered zero-, one-, two-, and three- body terms, respectively [28]. The normal ordered strings of creation and annihilation operators obey $\left\langle\Phi\left|\left\{a_{i}^{\dagger} \ldots a_{j}\right\}\right| \Phi\right\rangle=0$. Now, a continuous unitary transformation is applied to the Hamiltonian of Eq. 1. This unitary transformation is parameterized by a parameter $s$ which is called flow parameter:

$$
H(s)=U(s) H U^{\dagger}(s) \equiv H^{d}(s)+H^{o d}(s) .
$$

Here $H^{d}(s)$ is the diagonal part and $H^{o d}(s)$ is the off- diagonal part of the Hamiltonian. As $s \rightarrow \infty$, the offdiagonal matrix elements become zero.

The evolution of Hamiltonian with the flow parameter $s$ is given as:

$$
\frac{d H(s)}{d s}=[\eta(s), H(s)]
$$

where $\eta(s)$ is the anti-Hermitian generator of the unitary transformation given by

$$
\eta(s) \equiv \frac{d U(s)}{d s} U^{\dagger}(s)
$$

The $H^{o d}(s)$ permits us to decouple the $s d$ valence space from the core and higher shells as $s \rightarrow \infty$. The resulting Hamiltonian is used in the shell model calculations. In the present calculations, we use the effective interactions with $\hbar \Omega=24 \mathrm{MeV}$.

For the Hamiltonian of the CCEI approach, we have used the following $A$-dependent Hamiltonian as a starting point:

$$
\hat{H}_{A}=\sum_{i<j}\left(\frac{\left(\mathbf{p}_{i}-\mathbf{p}_{j}\right)^{2}}{2 m A}+\hat{V}_{N N}^{(i, j)}\right)+\sum_{i<j<k} \hat{V}_{3 \mathrm{~N}}^{(i, j, k)}
$$

The $N N$ and $3 N$ parts are taken from a next-to-nextto-next-to leading order $(N 3 L O)$ chiral nucleon-nucleon interaction, and a next-to-next-to leading order $(N 2 L O)$ chiral three-body interaction, respectively.

For both IM-SRG and CCEI, we use $\Lambda_{N N}=500 \mathrm{MeV}$ for the chiral $N 3 L O N N$ interaction $[45,46]$, and $\Lambda_{3 N}=$ $400 \mathrm{MeV}$ for the chiral N2LO $3 N$ interaction [47].

One can perform a unitary transformation of the Hamiltonian (5) to obtain the Hamiltonian used for the actual shell model calculations in the CCEI approach: 

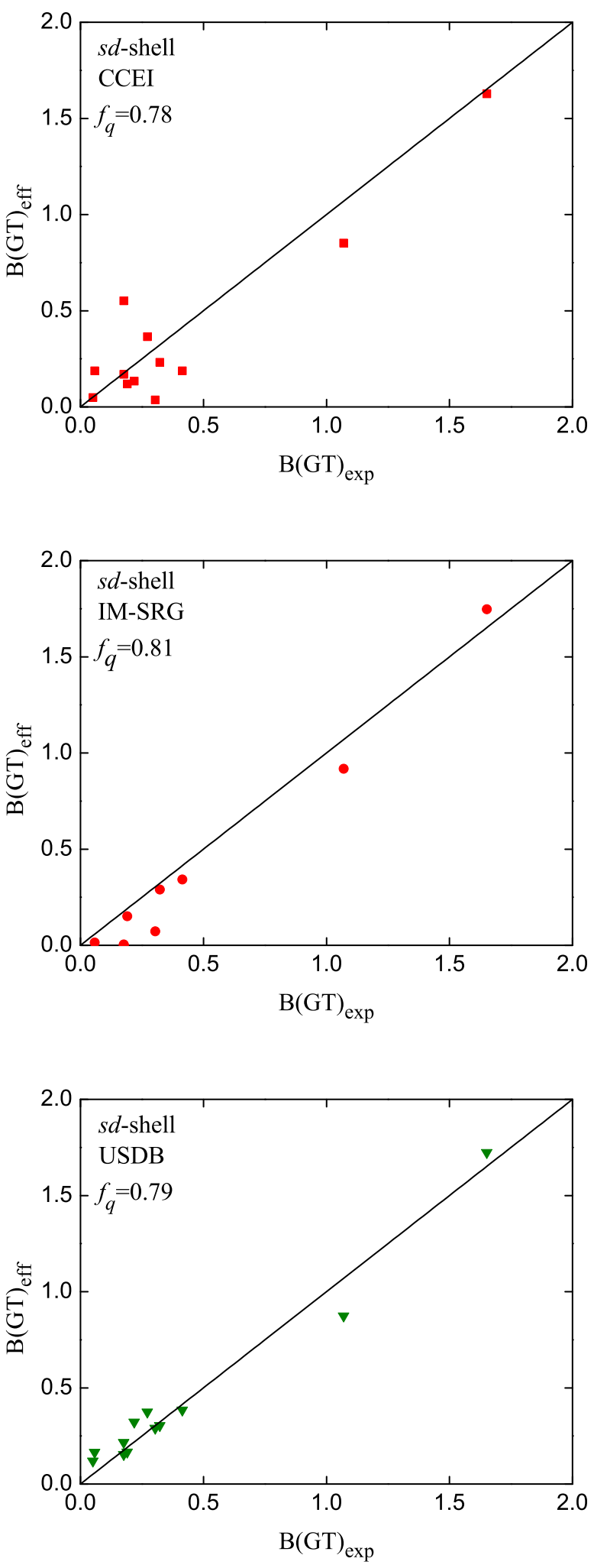

FIG. 1: The calculated value of quenching factor for GT transitions in $T=1 / 2 s d$ shell mirror nuclei with $A=17-39$ ( $A=17-33)$ using CCEI and USDB (IM-SRG) effective interactions.

$$
H_{C C E I}^{A}=H_{0}^{A_{c}}+H_{1}^{A_{c}+1}+H_{2}^{A_{c}+2}+\ldots
$$

Here the first term $H_{0}^{A_{c}}$ stands for the core, the second term $H_{1}^{A_{c}+1}$ for the valence one-body, and $H_{2}^{A_{c}+2}$ for the two- body Hamiltonian. The two-body term is derived from (5) by using Okubo-Lee-Suzuki (OLS) similarity transformation [48, 49]. By applying this unitary transformation, we get a non-Hermitian effective Hamiltonian. The similarity transformation is determined from the metric operator $\left[S^{\dagger} S\right]=P_{2}\left(1+\omega^{\dagger} \omega\right) P_{2}$ (see Ref. [50] for further details). Here, for making the Hamiltonian Hermitian, the metric operator $S^{\dagger} S$ is used. The Hermitian shell-model Hamiltonian is then obtained as $\left[S^{\dagger} S\right]^{1 / 2} \hat{H}_{C C E I}^{A}\left[S^{\dagger} S\right]^{-1 / 2}$.

In the present work two-body matrix elements for IMSRG and CCEI ab initio approaches have been adopted from Refs. [19] and [22], respectively.

The Gamow-Teller strength $B(G T)$ is calculated using the following expression,

$$
B\left(G T_{ \pm}\right)=\frac{1}{2 J_{i}+1} f_{q}^{2}\left|\left\langle f|| \sum_{k} \sigma^{k} \tau_{ \pm}^{k} \| i\right\rangle\right|^{2}
$$

where $\tau_{+}|p\rangle=|n\rangle, \tau_{-}|n\rangle=|p\rangle$, the index $k$ runs over the single particle orbitals, and $|i\rangle$ and $|f\rangle$ describe the state of the parent and daughter nuclei, respectively. In the present work we have taken the value of quenching factor as $f_{q}=0.77[51,52]$. In order to support our above choice, we show the calculated quenching factors for $T=1 / 2 s d$ shell nuclei with $A=17-39$ [53] using the three different interactions in Fig. 1. These quenching factors are obtained by a chi-square fit of the theoretical GT transition strengths to the corresponding experimental strengths. Note that the IM-SRG interaction and corresponding theoretical transition strengths are available up to $A=34$. We can see that the quenching factors obtained are 0.79, 0.78 and 0.81 for the USDB, CCEI and IM-SRG interactions, respectively. Although these values show a slight dependence on the interaction, in this work we adopt $f_{q}=0.77$ for all three interactions, as this is more consistent with the value $f_{q}=0.764 \pm 0.013$ obtained for USDB in ref. [52], where more data have been used for the fitting. With this choice $f_{q}=0.77$, the r.m.s. deviations from the experimental values are $0.088,0.177$ and 0.149 for USDB, CCEI and IM-SRG interactions, respectively. Compared to the USDB case, the enhancement of the r.m.s. deviations for CCEI and IM-SRG is qualitatively similar to the deviations for the energy levels $[19,22]$. In the case of IM-SRG, the calculated $B(G T)$ values are very small and the deviations from the experimental values become large for higher mass nuclei with $A \geq 27$. In the case of CCEI, large deviations are also seen in several nuclei with higher mass, $A=31,27,25$ and 33 with descending order of magnitude. This tendency may be attributed to the increasing number of 3 -valence nucleon combinations interacting via $3 N$ forces[19], which we neglected in our calculation. 
In the present work we have also checked the Ikeda sum rule $\left(B\left(G T_{-}\right)-B\left(G T_{+}\right)=3(N-Z)\right)$ for $A=23,24$ and 26. Both $a b$ intio interactions used in the IM-SRG and CCEI methods satisfy this sum rule, as does the phenomenological USDB interaction. Thus we are confident that enough excited states are taken into account in the calculation of GT strengths for sd shell nuclei in the two $a b$ initio calculations.

\section{COMPARISON OF THE EXPERIMENTAL AND THEORETICAL GT STRENGTH DISTRIBUTIONS}

In this section we compare the theoretical results with the experimental data.

\section{A. $\quad{ }^{20} \mathrm{Ne} \rightarrow{ }^{20} \mathbf{F}$}

In Fig. 2, we compare the $B(G T)$ strength distribution obtained from the two ab initio effective interactions CCEI (Fig. 2 (b)) and IM-SRG (Fig. 2 (c)) and the phenomenological USDB (Fig. 2 (d)) interaction with the experimental data for the transition ${ }^{20} \mathrm{Ne} \rightarrow{ }^{20} \mathrm{~F}$. The $B(G T)$ values for these transitions are known from Ref. [29], where the data is obtained from the reaction ${ }^{20} \mathrm{Ne}(n, p){ }^{20} \mathrm{~F}$ up to the excitation energy $E_{x}=10 \mathrm{MeV}$ of ${ }^{20} \mathrm{~F}$. On the horizontal axis the excitation energies of different $1^{+}$states of ${ }^{20} \mathrm{~F}$ are shown. In the experimental data the $B(G T)$ strength is spread over a wide range of excitation energies of ${ }^{20} \mathrm{~F}$. The theoretical calculations for the $B(G T)$ strength have already been done [29] in the framework of the shell model using the universal $s d$ shell (SD) interaction of Wildenthal [54]. All three interactions used here give the strongest peak around the excitation energy $\sim 1 \mathrm{MeV}$, and other strong peaks are observed around excitation energies $\sim 4 \mathrm{MeV}$ and $\sim 8$ $\mathrm{MeV}$. The other peaks are small in strength. In Fig. 2 (c), the strongest peak is observed around the excitation energy $\sim 1 \mathrm{MeV}$, but the strength is less than that of the strongest peak in the CCEI. The USDB interaction also shows the strongest peak at $\sim 1 \mathrm{MeV}$, but its strength is smaller than that obtained with both $a b$ initio interactions. In the experimental data shown in Fig. 2 (a), a wide spread of the $B(G T)$ strength distribution is observed in the energy range 6-7 MeV and 8-9 MeV. However, theoretically very small $B(G T)$ strength is obtained in the energy range 6-7 MeV. All three interactions also show zero strength in the energy range $2-3 \mathrm{MeV}$. From Fig. 2(b), 2(c) and 2(d), it is clear that, as we go towards higher excitation energy, the $B(G T)$ strength decreases and then increases again at the excitation energy around 8-9 MeV. All three interactions give the ground state (g.s.) $2^{+}$for ${ }^{20} \mathrm{~F}$ in agreement with the experiment. In Fig. 2(e), the accumulated sums of $B(G T)$ are shown as a function of excitation energy of ${ }^{20} \mathrm{~F}$. The CCEI gives better results compared to the other interactions. The

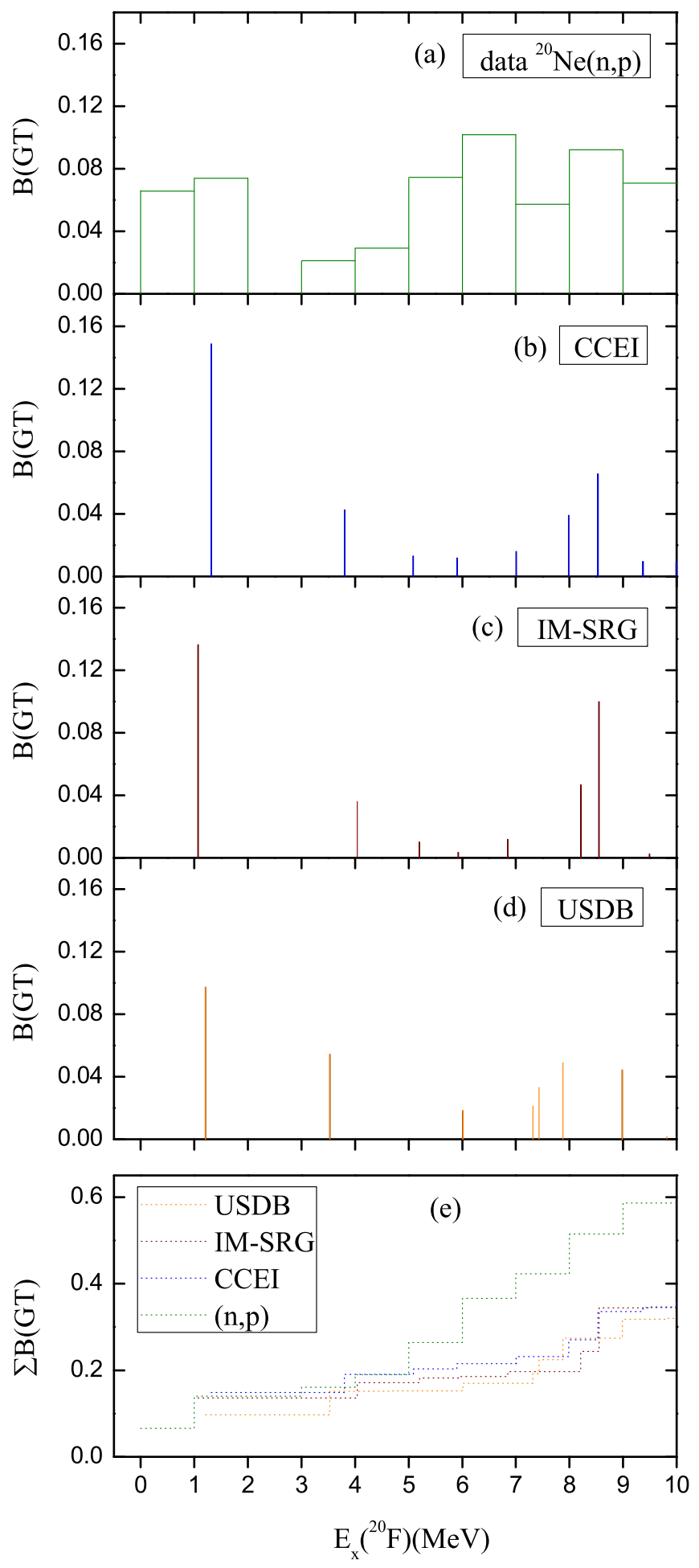

FIG. 2: Comparison of the experimental and theoretical $B(G T)$ distributions for ${ }^{20} \mathrm{Ne} \rightarrow{ }^{20} \mathrm{~F}$. 
summed $B(G T)$ values at higher excitation energies are lower than the experimental data for all three interactions used here. The small calculated $B(G T)$ values in comparison to the experimental values at $E_{x}>5 \mathrm{MeV}$ can be attributed to the limitation of the configuration space within the $s d$ shell. The breaking of ${ }^{16} \mathrm{O}$ core is important for the fragmentation of the GT strength. Shellmodel calculations can be performed in $p$-sd model space to include the $B(G T)$ strength beyond $E_{x}>10 \mathrm{MeV}$. The ${ }^{20} \mathrm{Ne}$ is a well deformed nucleus, and has admixtures of $g$-shell components [55]. We should also keep in mind that the experimental data have rather large errors, as large as 0.209 for the sum of $B(G T)$ [29].

\section{B. ${ }^{23} \mathrm{Na} \rightarrow{ }^{23} \mathrm{Mg}$}

Fig. 3 shows the experimental and theoretical $B(G T)$ strength distribution for the transition ${ }^{23} \mathrm{Na} \rightarrow{ }^{23} \mathrm{Mg}$. Here, the experimental data is available from the ${ }^{23} \mathrm{Na}\left({ }^{3} \mathrm{He}, t\right){ }^{23} \mathrm{Mg}$ reaction [30]. In this reaction the $B(G T)$ transitions were measured at the incident energy of $140 \mathrm{MeV}$ per nucleon with the energy resolution of 45 $\mathrm{keV}$. The ${ }^{23} \mathrm{Na}$ and ${ }^{23} \mathrm{Mg}$ are deformed nuclei with the static quadrupole moments $10.1 \pm 0.2 \mathrm{fm}^{2}$ [56] and 11.4 $\pm 0.3 \mathrm{fm}^{2}$ [57], respectively. These nuclei are important from astrophysical point of view. In Fig. 3 (a) the experimental data are shown up to the excitation energy $11.132 \mathrm{MeV}$ of ${ }^{23} \mathrm{Mg}$. In Ref. [30], the strength of the first transition $\left({ }^{23} \mathrm{Na}\left(\frac{3}{2}^{+}\right) \rightarrow{ }^{23} \mathrm{Mg}\left(\frac{3}{2}^{+}\right)\right)$is 0.340 , which is the mixture of $B(G T)$ and Fermi transition strength. We have excluded the Fermi transition strength. Now, the $B(G T)$ transition strength is 0.09 . The largest $B(G T)$ strength is at the excitation energy $8.168 \mathrm{MeV}$. Fig. 3 (b) shows the $B(G T)$ strength obtained in the CCEI. The CCEI gives a strong peak at the excitation energy $5.637 \mathrm{MeV}$, and the magnitude of strength is also comparable with the strongest peak observed in the experiment. This peak comes from the transition ${ }^{23} \mathrm{Na}\left(\frac{3}{2}_{1}^{+}\right) \rightarrow$ ${ }^{23} \mathrm{Mg}\left(\frac{5}{2}_{4}^{+}\right)$. In the energy range $6-12 \mathrm{MeV}$, we see many $B(G T)$ transitions which are not observed in the experiment. Fig. 3 (c) shows results of the IM-SRG interaction. The IM-SRG interaction gives the strongest peak of the $B(G T)$ strength at the excitation energy $5.826 \mathrm{MeV}$, and its strength is comparable with the strength of the strongest peak obtained with the CCEI and observed in the experiment. The density of peaks is smaller for the IM-SRG interaction compared to the CCEI. In Fig. 3 (d), we see the strongest peak at the excitation energy 8.513 $\mathrm{MeV}$. All three interactions give the g.s. $\frac{3}{2}^{+}$for ${ }^{23} \mathrm{Mg}$, in agreement with the experimentally observed g.s. The distribution of accumulated sums of $B(G T)$ for the experimental data and the theoretical calculations is shown in Fig. 3 (e). The CCEI and USDB interactions show similar trends for the summed $B(G T)$. The IM-SRG interaction gives larger values for the summed $B(G T)$ strengths than the CCEI or the experiment.

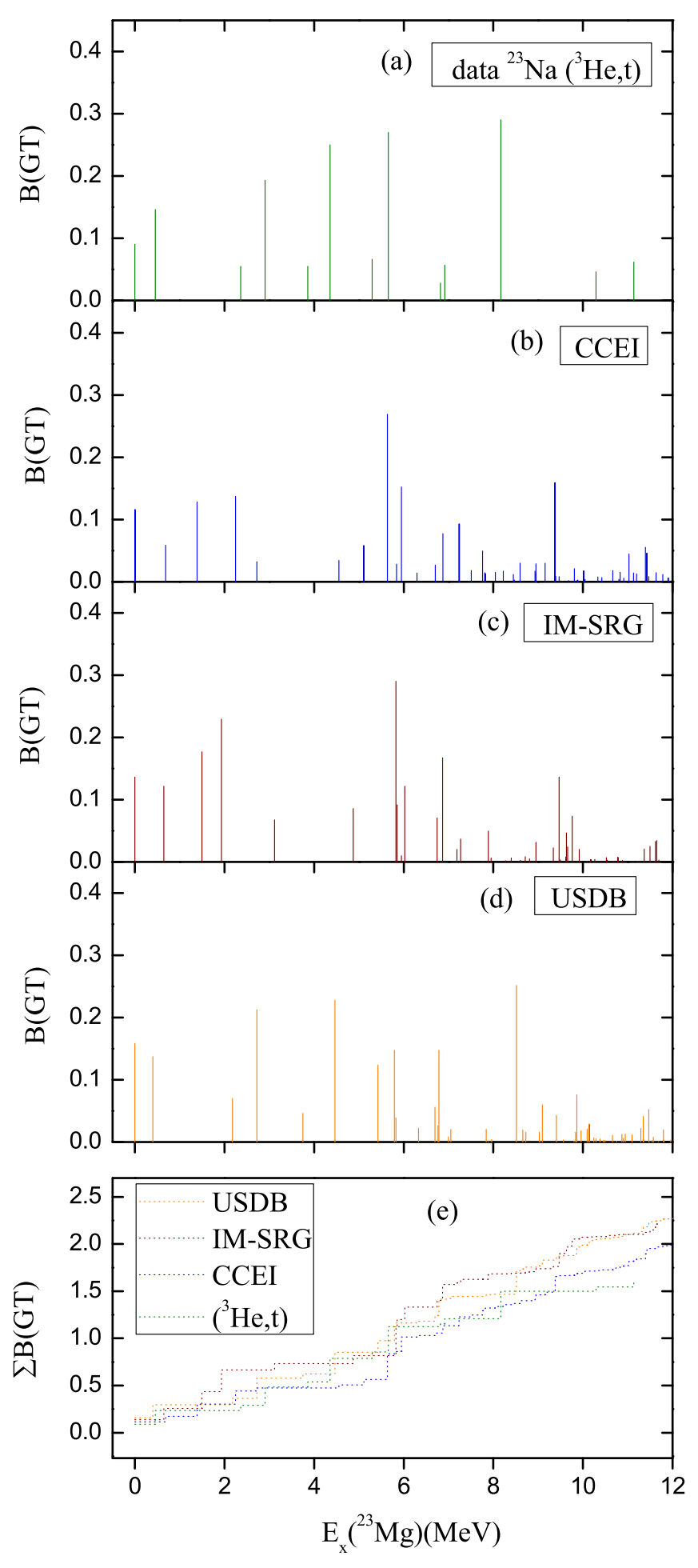

FIG. 3: Comparison of the experimental and theoretical $B(G T)$ distributions for ${ }^{23} \mathrm{Na} \rightarrow{ }^{23} \mathrm{Mg}$. 


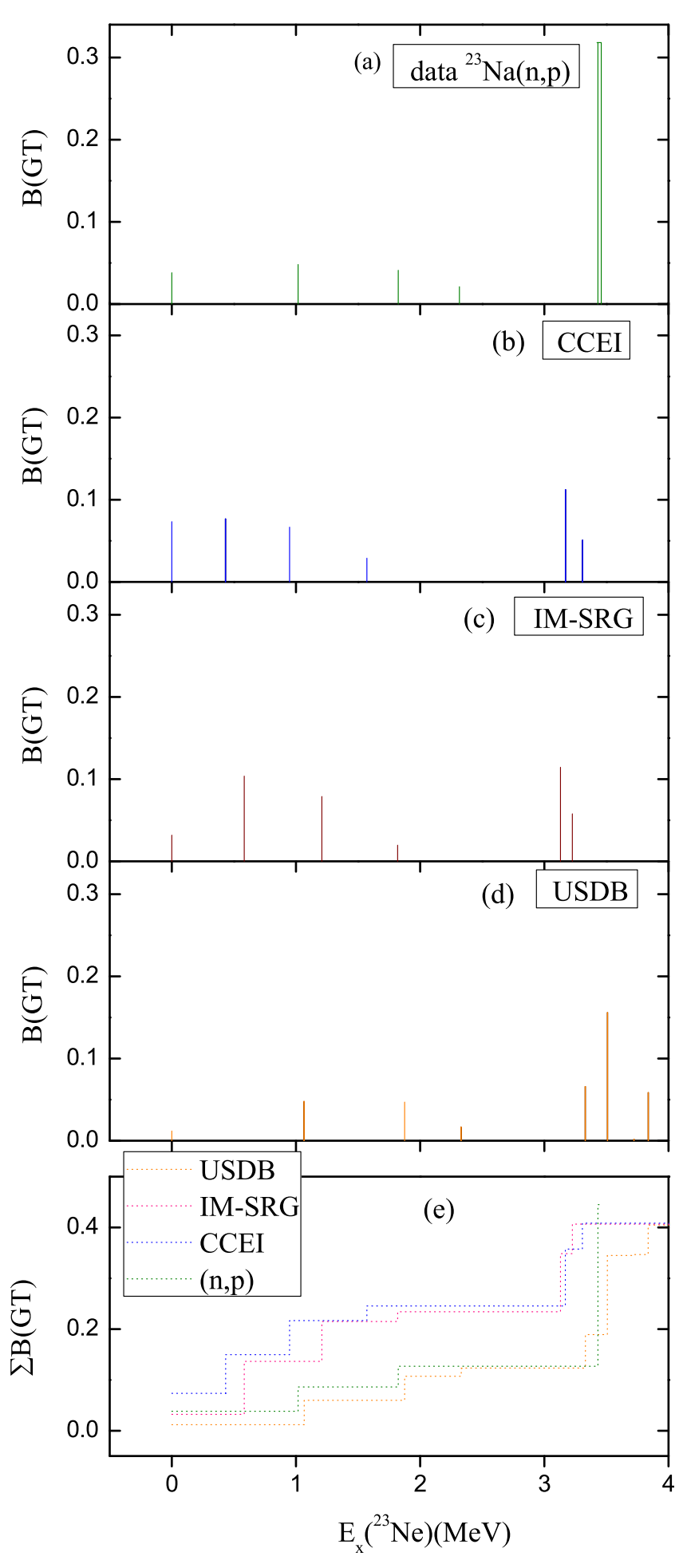

FIG. 4: Comparison of the experimental and theoretical $B(G T)$ distributions for ${ }^{23} \mathrm{Na} \rightarrow{ }^{23} \mathrm{Ne}$.

$$
\text { C. }{ }^{23} \mathrm{Na} \rightarrow{ }^{23} \mathrm{Ne}
$$

In Fig. 4 (a) the experimental $B(G T)$ strength distribution for the transition ${ }^{23} \mathrm{Na} \rightarrow{ }^{23} \mathrm{Ne}$ [31], observed in the charge exchange reaction ${ }^{23} \mathrm{Na}(n, p){ }^{23} \mathrm{Ne}$, is shown. Previously, the shell model results for $B(G T)$ distribution were shown in Ref. [31], and in the present work we show the calculations obtained by using the recent phenomenological USDB interaction in comparison with the $a b$ initio effective interactions. The experimental $B(G T)$ strength (Fig. 4 (a)) is dominated in the excitation energy range $3.432-3.458 \mathrm{MeV}$. The other peaks outside this range have very small $B(G T)$ values. With the CCEI (Fig. 4 (b)), we get the strongest peak at the excitation energy $3.170 \mathrm{MeV}$, but its strength is approximately three times less than the experimental value. We also see some other peaks with $B(G T)$ values below 0.1 . In the IM-SRG approach (4 (c)), we get two peaks with strengths larger than 0.1. In Fig. 4 (d), the $B(G T)$ distribution is shown for the USDB interaction. We see the strongest peak at the excitation energy $3.508 \mathrm{MeV}$. All strong peaks in the theoretical calculations correspond to the transition ${ }^{23} \mathrm{Na}\left(\frac{3}{2}_{1}^{+}\right) \rightarrow{ }^{23} \mathrm{Ne}\left(\frac{1}{2}_{2}^{+}\right)$. All three interactions give the g.s. $\frac{5}{2}^{+}$for ${ }^{23} \mathrm{Ne}$, in agreement with the experiment. The summed $B(G T)$ values (Fig. 4 (e)) obtained with USDB show a similar trend as the experimental values up to the excitation energy $3.5 \mathrm{MeV}$. The theoretical $B(G T)$ strength is generally lower than the experimental one.

$$
\text { D. }{ }^{24} \mathrm{Mg} \rightarrow{ }^{24} \mathrm{Na}
$$

Figure 5 shows the $B(G T)$ strength distribution obtained from the shell model calculations, and the experimental data for the transition ${ }^{24} \mathrm{Mg} \rightarrow{ }^{24} \mathrm{Na}$. There are two experimental data for the $B(G T)$ distribution available from the ${ }^{24} \mathrm{Mg}\left(t,{ }^{3} \mathrm{He}\right){ }^{24} \mathrm{Na}$ and ${ }^{24} \mathrm{Mg}\left(d,{ }^{2} \mathrm{He}\right){ }^{24} \mathrm{Na}$ reactions. The ${ }^{24} \mathrm{Mg}\left(t,{ }^{3} \mathrm{He}\right)^{24} \mathrm{Na}$ reaction was performed at the energy of $115 \mathrm{MeV}$ per nucleon, using a secondary triton beam with the energy resolution of about $200 \mathrm{keV}$ [32]. The ${ }^{24} \mathrm{Mg}\left(d,{ }^{2} \mathrm{He}\right)^{24} \mathrm{Na}$ reaction was performed at the energy $170 \mathrm{MeV}$, and a good resolution of the order of $145 \mathrm{keV}$ was obtained in this reaction [33]. The shell model calculations with the phenomenological interactions USDA and USDB have already been performed [32]. Fig. 5 (a) shows the data from ${ }^{24} \mathrm{Mg}\left(t,{ }^{3} \mathrm{He}\right)^{24} \mathrm{Na}$ reaction. In this case, we see the strongest peak at the excitation energy $1.346 \mathrm{MeV}$ of ${ }^{24} \mathrm{Na}$. We also find that the distribution of the $B(G T)$ strength is in the energy windows from 3.14-3.94 MeV and 6.5-7.1 MeV for this reaction. Fig. 5 (b) shows the experimental information for the $B(G T)$ distribution from ${ }^{24} \mathrm{Mg}\left(d,{ }^{2} \mathrm{He}\right)^{24} \mathrm{Na}$ reaction. This reaction gives the strongest peak at the excitation energy $1.35 \mathrm{MeV}$ with nearly the same $B(G T)$ value as obtained from the ${ }^{24} \mathrm{Mg}\left(t,{ }^{3} \mathrm{He}\right){ }^{24} \mathrm{Na}$ reaction. Some other peaks are also observed with less strength. Fig. 


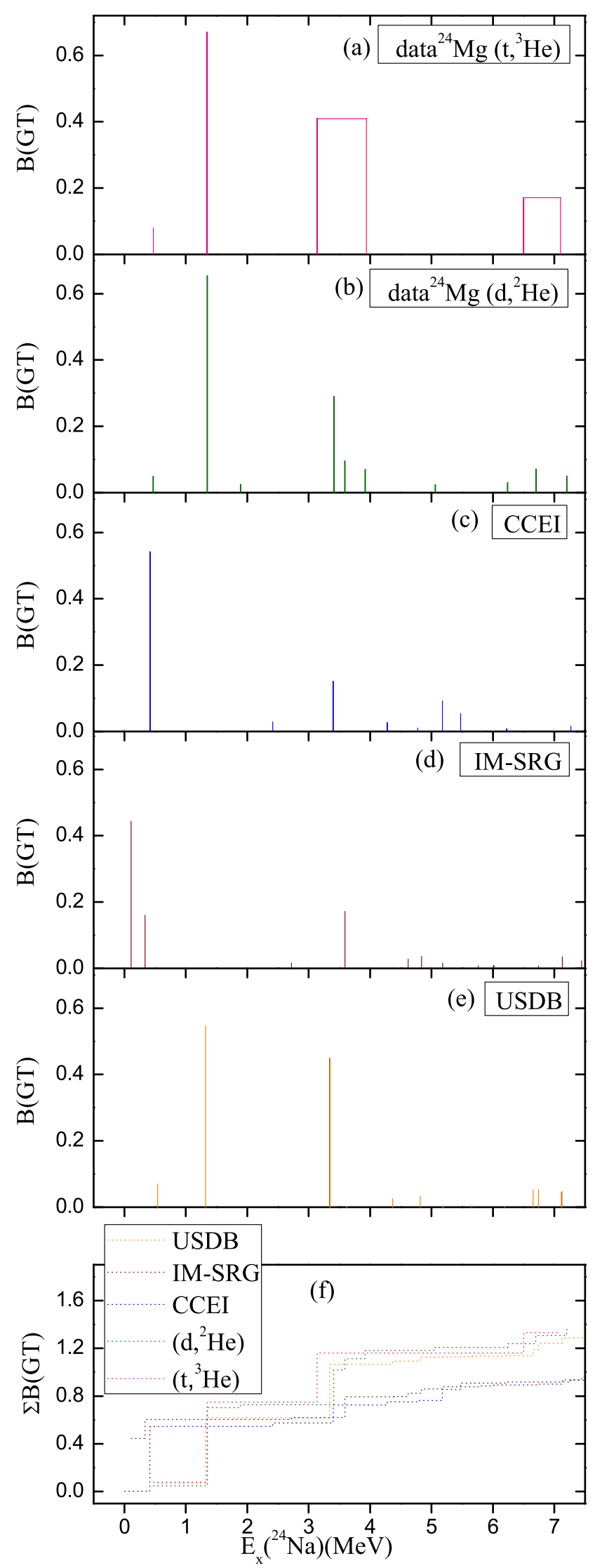

5 (c) shows the $B(G T)$ distribution obtained with the CCEI. The CCEI predicts $1^{+}$as the g.s. of ${ }^{24} \mathrm{Na}$, while the g.s. from the experiment is $4^{+}$. In Fig. 5 (c) we see the strongest peak at the excitation energy $0.417 \mathrm{MeV}$, which comes from the transition ${ }^{24} \mathrm{Mg}\left(0^{+}\right) \rightarrow{ }^{24} \mathrm{Na}\left(1_{2}^{+}\right)$. The $B(G T)$ distribution obtained with the IM-SRG interaction is shown in Fig. 5 (d). The IM-SRG interaction gives the g.s. of ${ }^{24} \mathrm{Na}$ as $2^{+}$. With this interaction we get the strongest peak at the excitation energy $0.110 \mathrm{MeV}$, which comes from the transition ${ }^{24} \mathrm{Mg}\left(0^{+}\right) \rightarrow{ }^{24} \mathrm{Na}\left(1_{1}^{+}\right)$. Fig. 5 (e) shows the $B(G T)$ distribution obtained with the phenomenological USDB interaction. Here we see the strongest peak at the excitation energy $1.323 \mathrm{MeV}$ and the next strongest peak at $3.345 \mathrm{MeV}$, which come from the transitions ${ }^{24} \mathrm{Mg}\left(0^{+}\right) \rightarrow{ }^{24} \mathrm{Na}\left(1_{2}^{+}\right)$and ${ }^{24} \mathrm{Mg}\left(0^{+}\right) \rightarrow$ ${ }^{24} \mathrm{Na}\left(1_{3}^{+}\right)$, respectively. Fig. 5 (f) shows the trend of the accumulated sums of the $B(G T)$ distribution obtained from the experimental data and the theoretical calculations. The USDB interaction gives a similar trend as the experimental data for both reactions.

$$
\text { E. }{ }^{24} \mathrm{Mg} \rightarrow{ }^{24} \mathrm{Al}
$$

The experimental information on the $B(G T)$ strength distribution for the transition ${ }^{24} \mathrm{Mg} \rightarrow{ }^{24} \mathrm{Al}$ is available from the ${ }^{24} \mathrm{Mg}\left({ }^{3} \mathrm{He}, t\right){ }^{24} \mathrm{Al}$ reaction observed at $420 \mathrm{MeV}$ [35], and the ${ }^{24} \mathrm{Mg}(p, n){ }^{24} \mathrm{Al}$ reaction observed at 136 $\mathrm{MeV}$ [34]. The results of the shell model calculation for the $B(G T)$ strength have been previously reported in Ref. [35], where the phenomenological interactions USDA and USDB were employed. Fig. 6 (a) shows the data from the ${ }^{24} \mathrm{Mg}\left({ }^{3} \mathrm{He}, t\right){ }^{24} \mathrm{Al}$ reaction. We see the strongest peak at the excitation energy $1.090 \mathrm{MeV}$, and the next strongest peak at $3.001 \mathrm{MeV}$. Other peaks are also observed with less strengths. Fig. 6 (b) shows the data from the ${ }^{24} \mathrm{Mg}(p, n)^{24} \mathrm{Al}$ reaction. In this reaction the strongest peak is observed at the excitation energy $1.07 \mathrm{MeV}$, and the next strongest peak at $2.98 \mathrm{MeV}$. In Fig. 6 (c) the theoretical $B(G T)$ distribution, obtained by using the CCEI, is shown. The CCEI gives the g.s. $2^{+}$for ${ }^{24} \mathrm{Al}$, whereas the experimental g.s. is $4^{+}$. The CCEI gives the strongest peak at the excitation energy $0.615 \mathrm{MeV}$, which comes from the transition ${ }^{24} \mathrm{Mg}\left(0^{+}\right) \rightarrow$ ${ }^{24} \mathrm{Al}\left(1_{2}^{+}\right)$. The second strongest peak is observed at the excitation energy $3.205 \mathrm{MeV}$. In Fig. 6 (d) the theoretical $B(G T)$ distribution is shown with the IM-SRG interaction. The IM-SRG interaction gives g.s. as $2^{+}$ for ${ }^{24} \mathrm{Al}$. The IM-SRG interaction gives the strongest peak at the excitation energy $0.077 \mathrm{MeV}$ which comes from the transition ${ }^{24} \mathrm{Mg}\left(0^{+}\right) \rightarrow{ }^{24} \mathrm{Al}\left(1_{1}^{+}\right)$. The second strongest peak is observed at the excitation energy 3.486 MeV. Fig. 6 (e) shows the theoretical $B(G T)$ distribution obtained with the USDB interaction. The USDB interaction gives the strongest peak at $0.783 \mathrm{MeV}$ which comes from the transition ${ }^{24} \mathrm{Mg}\left(0^{+}\right) \rightarrow{ }^{24} \mathrm{Al}\left(1_{2}^{+}\right)$, and the second strongest peak at $2.805 \mathrm{MeV}$. The USDB interaction gives the g.s. $4^{+}$for ${ }^{24} \mathrm{Al}$, in agreement with

FIG. 5: Comparison of the experimental and theoretical $B(G T)$ distributions for ${ }^{24} \mathrm{Mg} \rightarrow{ }^{24} \mathrm{Na}$. 


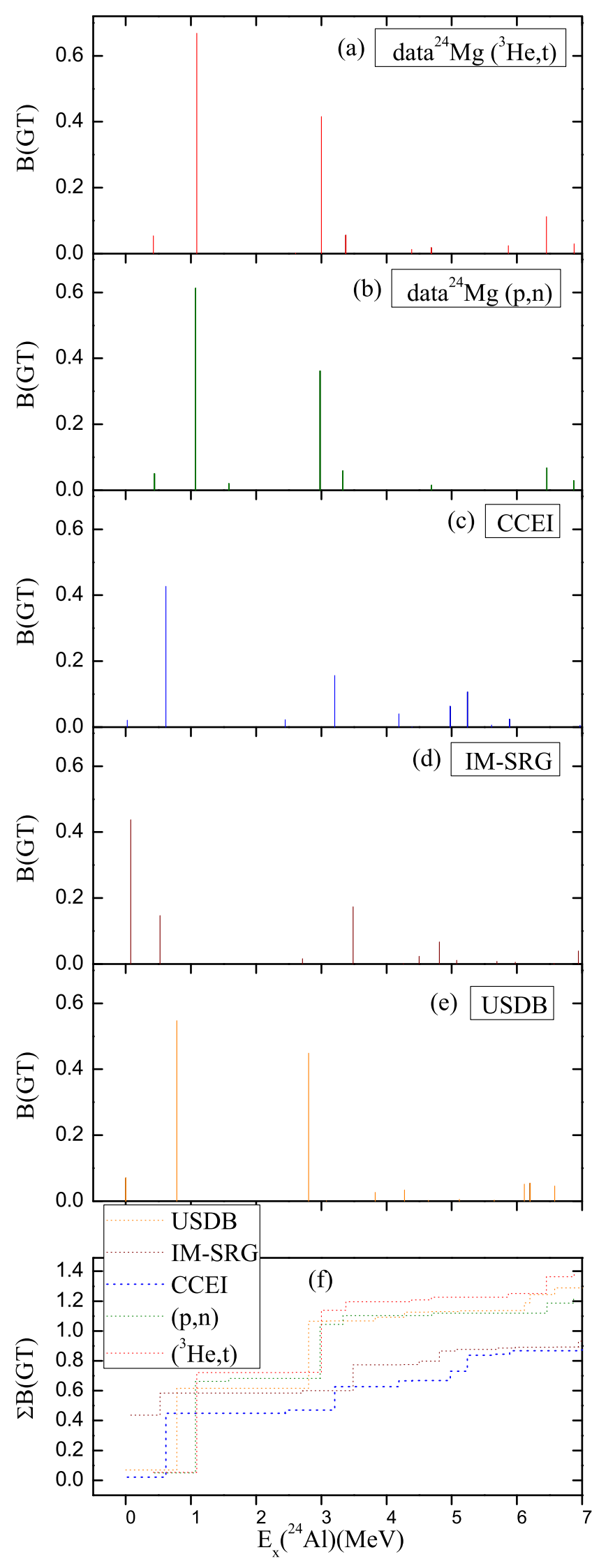

the experiment. The summed $B(G T)$ strength distribution is shown up to the excitation energy $7 \mathrm{MeV}$. The USDB interaction gives a reasonable agreement with the experimental data, while the results from ab initio interactions show smaller strength in comparison with the experiment.

$$
\text { F. }{ }^{25} \mathrm{Mg} \rightarrow{ }^{25} \mathrm{Al}
$$

The $B(G T)$ strength distribution for the transition ${ }^{25} \mathrm{Mg} \rightarrow{ }^{25} \mathrm{Al}$ is shown in Fig. 7 (a). This distribution has been measured via the ${ }^{25} \mathrm{Mg}\left({ }^{3} \mathrm{He}, t\right){ }^{25} \mathrm{Al}$ reaction at the energy of $140 \mathrm{MeV}$ per nucleon [36]. The ${ }^{25} \mathrm{Mg}$ and ${ }^{25} \mathrm{Al}$ nuclei are known to be strongly deformed, and the states of these mirror nuclei are well described in terms of the particle rotor model [36]. In the experiment, the $B(G T)$ strength from the transition ${ }^{25} \mathrm{Mg}\left(\frac{5}{2}_{1}^{+}\right)$to ${ }^{25} \mathrm{Al}\left(\frac{5}{2}_{1}^{+}\right)$is dominant, while the other $B(G T)$ strengths are very much suppressed. The explanation of the suppression of $B(G T)$ transitions in $A=25$ system is given on the basis of the selection rules of the $K$ quantum number in rotational bands, and also assuming the usual selection rule $\triangle J^{\pi}=1^{+}$for the $B(G T)$ operator. Most of the observed $B(G T)$ transition strength is very small and less reliable, see Ref. [36]. In particular, it is very weak in the $\sim 2$ - $6 \mathrm{MeV}$ energy range. In Fig. 7 (b), which shows the theoretical results obtained by using the CCEI approach, we see a considerable amount of $B(G T)$ strength in the 2 - $6 \mathrm{MeV}$ energy range. This method gives two dominant peaks at excitation energies $0.474 \mathrm{MeV}$ and 1.847 $\mathrm{MeV}$ with smaller $B(G T)$ values than the experiment. The first peak comes from the transition ${ }^{25} \mathrm{Mg}\left(\frac{5}{2}_{1}^{+}\right)$to ${ }^{25} \mathrm{Al}\left(\frac{5}{2}_{1}^{+}\right)$, and the second one comes from ${ }^{25} \mathrm{Mg}\left(\frac{5}{2}_{1}^{+}\right)$to ${ }^{25} \mathrm{Al}\left(\frac{7}{2}_{1}^{+}\right)$. In Fig. 7 (c), which shows the results for the IM-SRG, we see a peak as in the experiment around $\sim 6$ $\mathrm{MeV}$. The strength distribution calculated with the IMSRG interaction gives the first dominant peak at higher energy in comparison with the experiment. Fig. 7 (d) shows the $B(G T)$ strength distribution obtained with the USDB interaction. The first two peaks show a reasonable agreement with experiment. Above the excitation energy $6 \mathrm{MeV}$, we see a peak with smaller magnitude in comparison with experiment. The accumulated sums from the theoretical calculations and experimental data are shown in Fig. 7 (e). The USDB results agree reasonably well with experiment, compared to the $a b$ initio interactions.

$$
\text { G. }{ }^{26} \mathrm{Mg} \rightarrow{ }^{26} \mathrm{Na}
$$

The $B(G T)$ strength distribution for the transition ${ }^{26} \mathrm{Mg} \rightarrow{ }^{26} \mathrm{Na}$ is shown in Fig. 8. Fig. 8 (a) shows the distribution observed with the ${ }^{26} \mathrm{Mg}\left(t,{ }^{3} \mathrm{He}\right){ }^{26} \mathrm{Na}$ reaction at the energy of $115 \mathrm{MeV}$ per nucleon [37]. From the experimental data ${ }^{26} \mathrm{Mg}\left(t,{ }^{3} \mathrm{He}\right){ }^{26} \mathrm{Na}$, we see the most intense peak at the excitation energy $0.08 \mathrm{MeV}$

FIG. 6: Comparison of the experimental and theoretical $B(G T)$ distributions for ${ }^{24} \mathrm{Mg} \rightarrow{ }^{24} \mathrm{Al}$. 


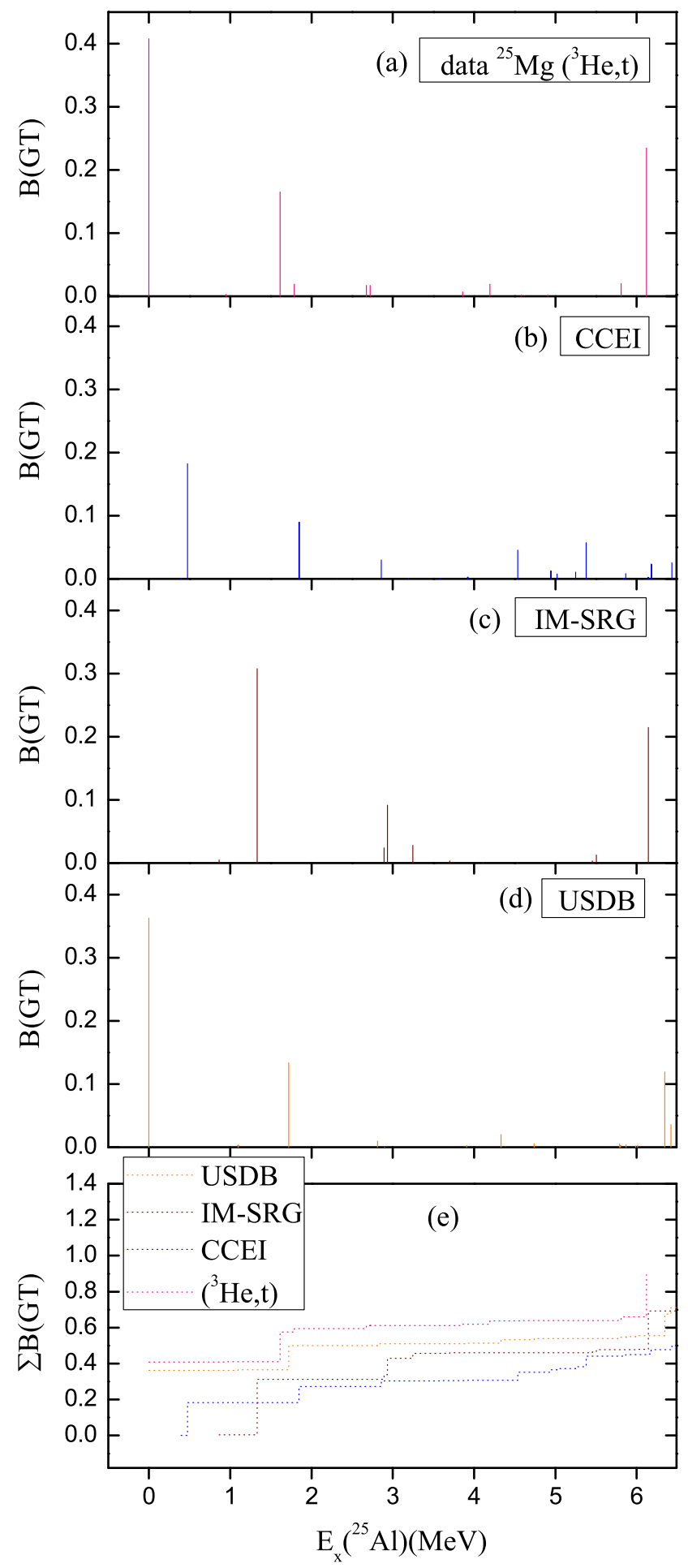

FIG. 7: Comparison of the experimental and theoretical $B(G T)$ distributions for ${ }^{25} \mathrm{Mg} \rightarrow{ }^{25} \mathrm{Al}$.

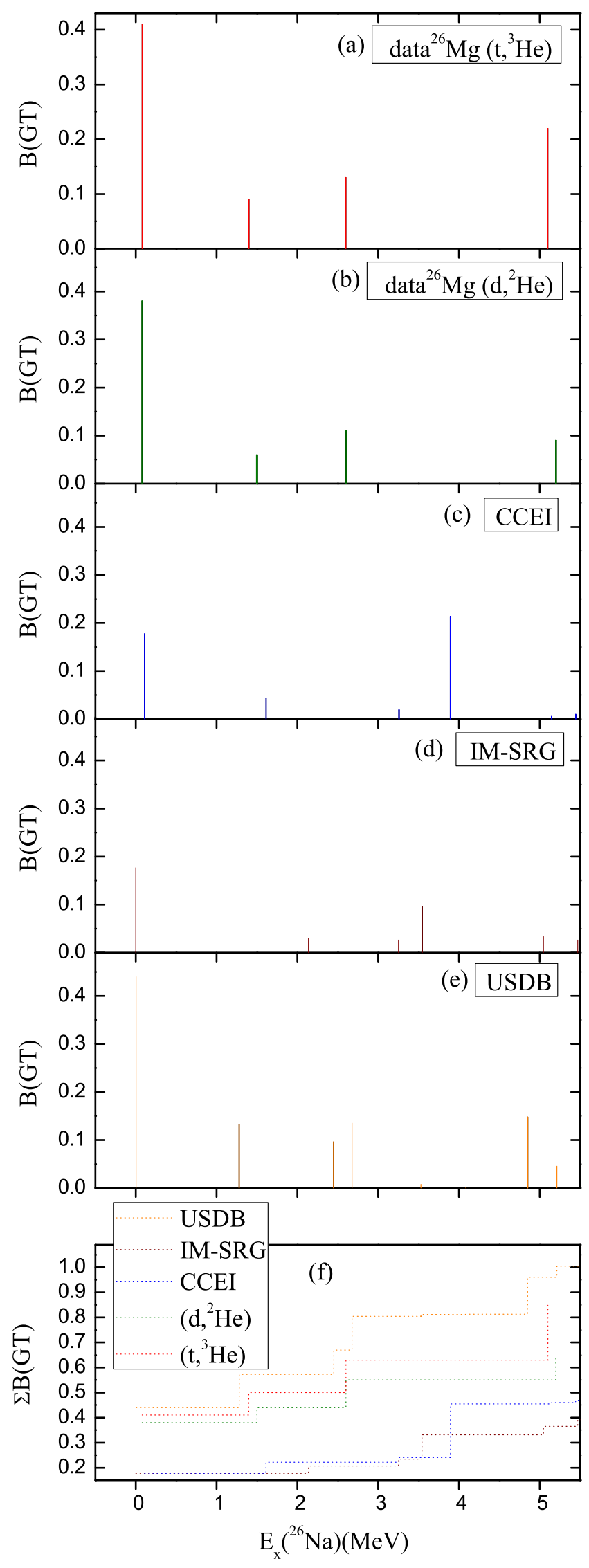

FIG. 8: Comparison of the experimental and theoretical $B(G T)$ distributions for ${ }^{26} \mathrm{Mg} \rightarrow{ }^{26} \mathrm{Na}$. 
of ${ }^{26} \mathrm{Na}$. Fig. 8 (b) shows the experimental information from the ${ }^{26} \mathrm{Mg}\left(d,{ }^{2} \mathrm{He}\right)^{26} \mathrm{Na}$ reaction. This reaction also shows a strong peak at the excitation energy 0.08 $\mathrm{MeV}$. The $B(G T)$ strenghts from the ${ }^{26} \mathrm{Mg}\left(d,{ }^{2} \mathrm{He}\right){ }^{26} \mathrm{Na}$ data are smaller in magnitude than those from the ${ }^{26} \mathrm{Mg}\left(t,{ }^{3} \mathrm{He}\right){ }^{26} \mathrm{Na}$ data. Fig. 8 (c) shows the distribution obtained with the CCEI approach. This method gives the g.s. of ${ }^{26} \mathrm{Na}$ as $2^{+}$, whereas the experimental g.s. is $3^{+}$. With the CCEI, a strong peak is observed at the excitation energy $3.894 \mathrm{MeV}$. Fig. 8 (d) shows the theoretical calculations obtained with the IM-SRG interaction. From this figure we see the strongest peak at zero excitation energy, but the strength of this transition is less than half of the strength observed for the strongest peak in the experiment. Other calculated strengths are also weak in comparison with both the experimental data. The IMSRG interaction gives $1^{+}$as the g.s. of ${ }^{26} \mathrm{Na}$. Fig. 8 (e) shows the distribution obtained with the USDB interaction. It shows a strong peak which is comparable with both experimental data. The USDB interaction gives $3^{+}$as the g.s. of ${ }^{26} \mathrm{Na}$, in agreement with experiment. The accumulated sums are shown in Fig. 8 (f). The results obatined from the USDB interaction are much better than the $a b$ initio interactions.

\section{H. $\quad{ }^{26} \mathrm{Mg} \rightarrow{ }^{26} \mathrm{Al}$}

Fig. 9 shows the GT strength distribution for the transition from ${ }^{26} \mathrm{Mg} \rightarrow{ }^{26} \mathrm{Al}$. Information on the $B(G T)$ strength is available from the ${ }^{26} \mathrm{Mg}\left({ }^{3} \mathrm{He}, t\right){ }^{26} \mathrm{Al}$ [37] (Fig. 9 (a)) and ${ }^{26} \mathrm{Mg}(p, n){ }^{26} \mathrm{Al}$ [39] (Fig. 9 (b)) reactions. The ${ }^{26} \mathrm{Mg}\left({ }^{3} \mathrm{He}, \mathrm{t}\right){ }^{26} \mathrm{Al}$ reaction was observed at $140 \mathrm{MeV} /$ nucleon with energy resolution of $100 \mathrm{keV}$. In Fig. 9 (c) the results obtained by using the CCEI are shown. In this case we see a strong transition at the excitation energy $3.498 \mathrm{MeV}$ of ${ }^{26} \mathrm{Al}$, which comes from the transition ${ }^{26} \mathrm{Mg}\left(0^{+}\right) \rightarrow{ }^{26} \mathrm{Al}\left(1_{5}^{+}\right)$. The CCEI gives the g.s. $3^{+}$for ${ }^{26} \mathrm{Al}$, while the experimental g.s. is $5^{+}$. Fig. 9 (d) shows the $B(G T)$ strength distribution using the IMSRG interaction. In this case we see a strong peak at the excitation energy $1.849 \mathrm{MeV}$, which comes from the transition ${ }^{26} \mathrm{Mg}\left(0^{+}\right) \rightarrow{ }^{26} \mathrm{Al}\left(1_{4}^{+}\right)$. The IM-SRG interaction gives g.s. $1^{+}$for ${ }^{26} \mathrm{Al}$. In Fig. 9 (e) the $B(G T)$ strength distribution obtained by using the phenomenological interaction USDB is shown. This interaction gives a strong peak at the excitation energy $1.034 \mathrm{MeV}$, which comes from the transition ${ }^{26} \mathrm{Mg}\left(0^{+}\right) \rightarrow{ }^{26} \mathrm{Al}\left(1_{1}^{+}\right)$. The USDB interaction gives $5^{+}$g.s. for ${ }^{26} \mathrm{Al}$, in agreement with the experiment. Fig. 9 (f) shows the accumulated sums of $B(G T)$ strength for the theoretical calculations and the experimental data. The IM-SRG and USDB interactions show almost the same trend as the experimental data, while the CCEI interaction gives lower values.

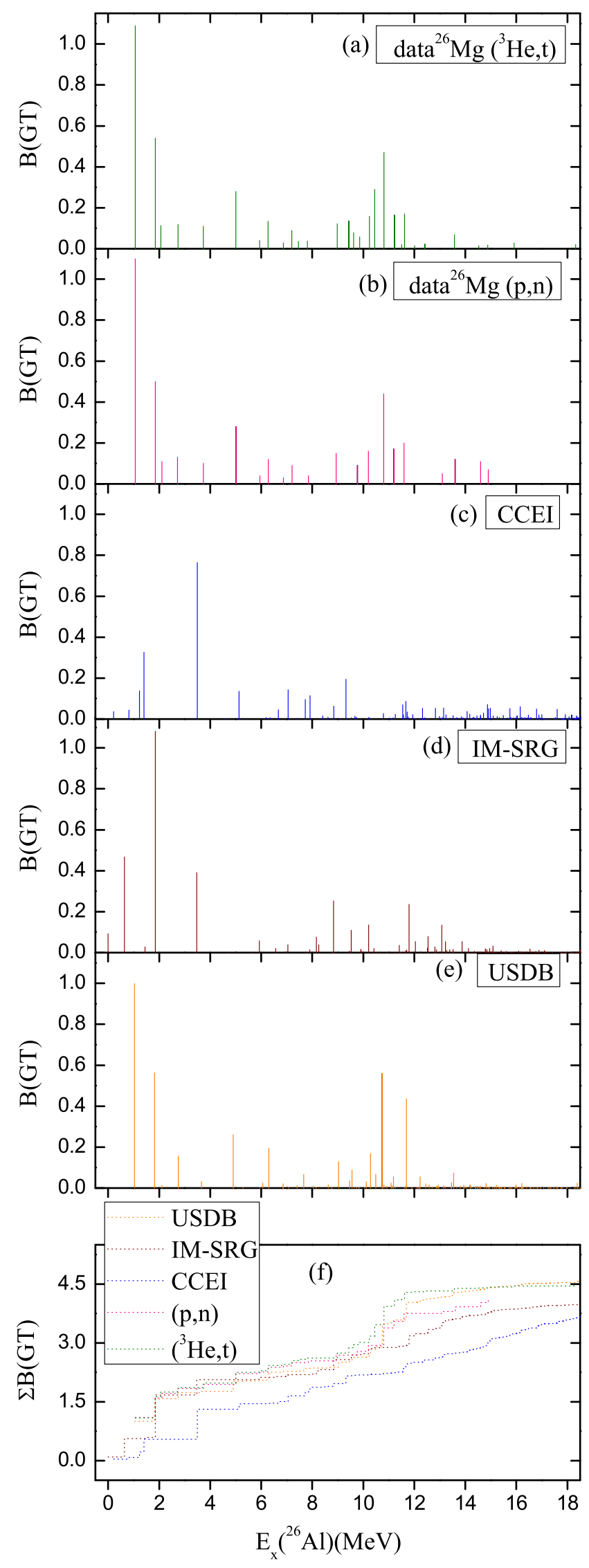

FIG. 9: Comparison of the experimental and theoretical $B(G T)$ distributions for ${ }^{26} \mathrm{Mg} \rightarrow{ }^{26} \mathrm{Al}$. 

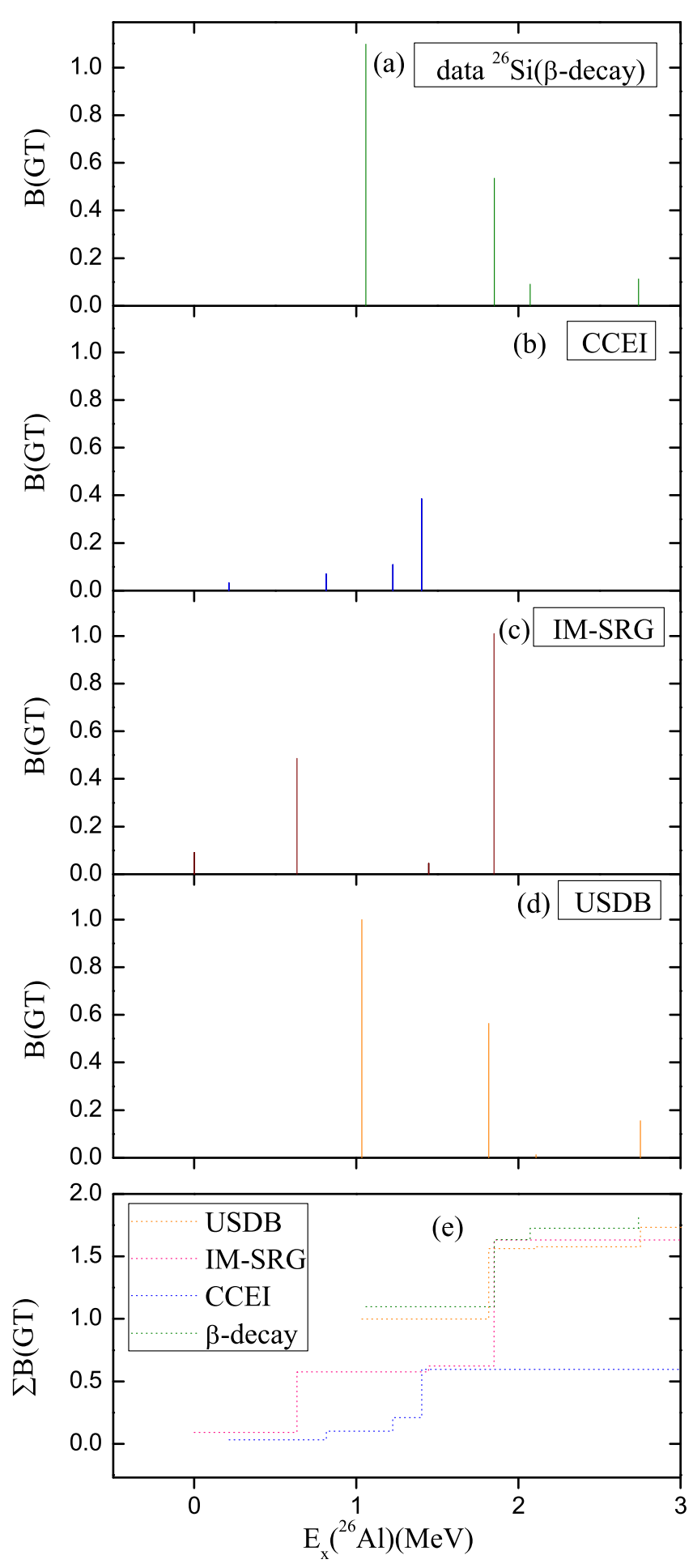

FIG. 10: Comparison of the experimental and theoretical $B(G T)$ distributions for ${ }^{26} \mathrm{Si} \rightarrow{ }^{26} \mathrm{Al}$.

$$
\text { I. }{ }^{26} \mathbf{S i} \rightarrow{ }^{26} \mathbf{A l}
$$

The experimental and theoretical information on the $B(G T)$ strength distribution for the transition ${ }^{26} \mathrm{Si} \rightarrow$ ${ }^{26} \mathrm{Al}$ is shown in Fig. 10. In Fig. 10 (a) the experimental data from $\beta$ - decay [40] is shown. We see a strong peak at the excitation energy $1.0577 \mathrm{MeV}$ of ${ }^{26} \mathrm{Al}$. The experimental data are very sparse. The only four peaks are observed up to the excitation energy $3 \mathrm{MeV}$ of ${ }^{26} \mathrm{Al}$. In Fig. $10(\mathrm{~b})$, the theoretical $B(G T)$ strength distribution obtained by using the CCEI is shown. It gives a strong peak at the excitation energy $1.403 \mathrm{MeV}$. The strength of this peak is smaller in comparison with the strongest peak from the experimental data. The CCEI gives $3^{+}$as the g.s. of ${ }^{26} \mathrm{Al}$, whereas the experimental g.s. is $5^{+}$. Fig. 10 (c) shows the $B(G T)$ strength distribution for the IMSRG interaction. We get a strong peak at the excitation energy $1.849 \mathrm{MeV}$. The strength of this peak is comparable with the experimental data. The IM-SRG interaction gives $1^{+}$g.s. for ${ }^{26} \mathrm{Al}$. Fig. $10(\mathrm{~d})$ shows the $B(G T)$ strength distribution from the USDB interaction. The USDB interaction gives a strong peak at the excitation energy $1.034 \mathrm{MeV}$. The results from the USDB interaction are in better agreement with the experimental data, compared to both $a b$ initio interactions. The USDB interaction gives $5^{+}$g.s. for ${ }^{26} \mathrm{Al}$, in agreement with the experiment. Fig. 10 (e) shows the accumulated sums of $B(G T)$ strengths for the experimental data and the theoretical calculations. The summed $B(G T)$ strength from the USDB interaction matches well with the experimental data, whereas the IM-SRG interaction shows the same trend after the excitation energy $2 \mathrm{MeV}$. The CCEI gives lower values in comparison with the experimental data.

$$
\text { J. } \quad{ }^{27} \mathrm{Al} \rightarrow{ }^{27} \mathrm{Si}
$$

The nuclei ${ }^{27} \mathrm{Si}$ and ${ }^{27} \mathrm{Al}$ are $T=1 / 2$ mirror nuclei. The information on the $B(G T)$ strength distribution for the transition ${ }^{27} \mathrm{Al} \rightarrow{ }^{27} \mathrm{Si}$ is given in Ref. [41]. For these transitions only one experimental data set is available from the reaction ${ }^{27} \mathrm{Al}\left({ }^{3} \mathrm{He}, t\right){ }^{27} \mathrm{Si}$, which was performed at $150 \mathrm{MeV} /$ nucleon and at scattering angle $0^{\circ}$. The $B(G T)$ strength distribution up to the excitation energy 9.95 MeV is shown in Fig. 11 (a). In the experimental data, the strength of the transition ${ }^{27} \mathrm{Al}\left(\frac{5}{2}_{1}^{+}\right) \rightarrow{ }^{27} \mathrm{Si}\left(\frac{5}{2}_{1}^{+}\right)$ is larger than the other $B(G T)$ strengths $\left(\right.$ At $E_{x}\left({ }^{27} \mathrm{Si}\right)=$ $0.0 \mathrm{MeV}$, this strength is obtained by removing the Fermi transition strength). The experimental $B(G T)$ strength at the excitation energies $0.98,5.51$ and $5.84 \mathrm{MeV}$ are not very reliable, and those at $4.49,5.30$ and $6.06 \mathrm{MeV}$ are less reliable [41]. Fig. 11 (b) shows the results obtained with the CCEI. At lower energies, the $B(G T)$ strength distribution is very small. We get a strong peak at the excitation energy $9.195 \mathrm{MeV}$ of ${ }^{27} \mathrm{Si}$, which comes from the transition ${ }^{27} \mathrm{Al}\left(\frac{5}{2}_{1}^{+}\right) \rightarrow{ }^{27} \operatorname{Si}\left(\frac{7}{2}_{12}^{+}\right)$. In the region of the 


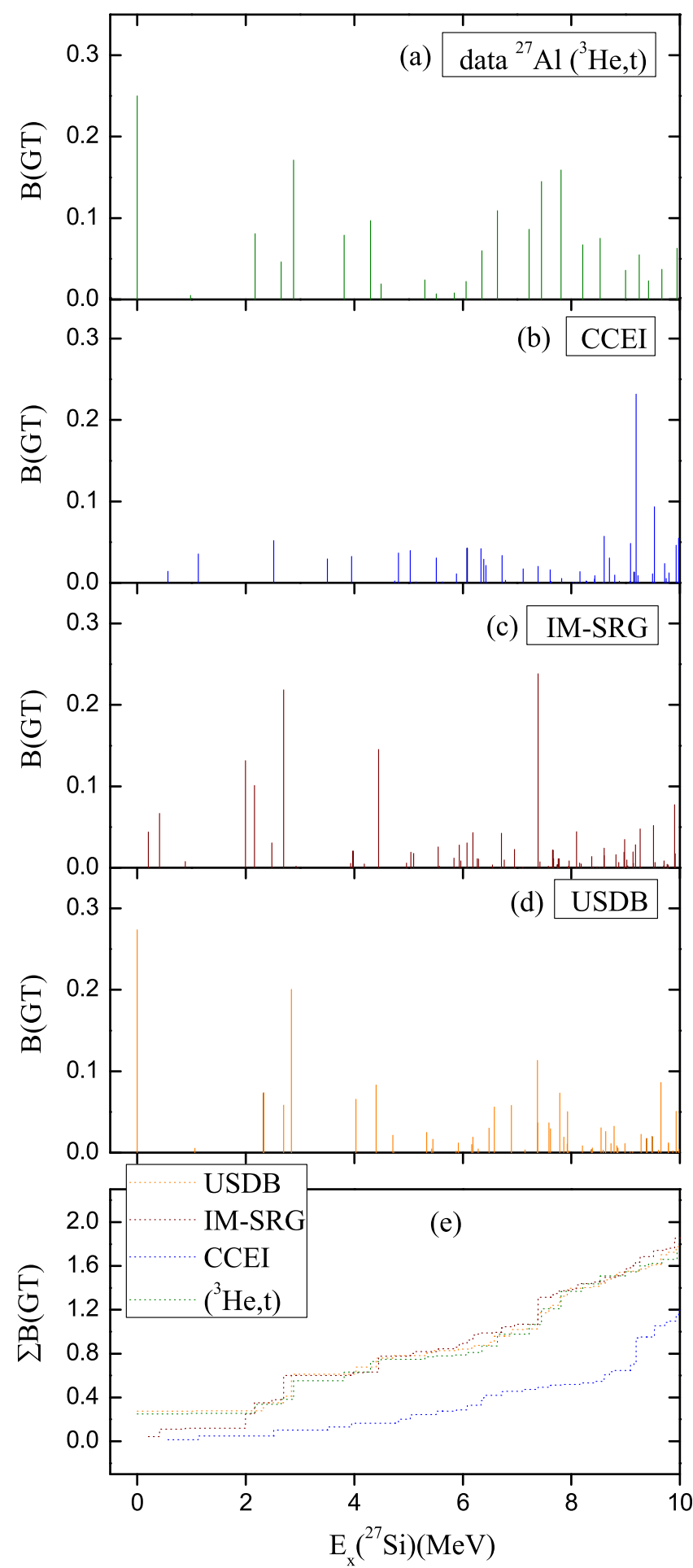

FIG. 11: Comparison of the experimental and theoretical $B(G T)$ distributions for ${ }^{27} \mathrm{Al} \rightarrow{ }^{27} \mathrm{Si}$. excitation energy range $5-10 \mathrm{MeV}$, the $B(G T)$ strengths are more dense as compared to below $5 \mathrm{MeV}$. For ${ }^{27} \mathrm{Si}$, the CCEI gives $\frac{3}{2}^{+}$as the g.s. of ${ }^{27} \mathrm{Si}$, while the experimental g.s. is $\frac{5}{2}^{+}$. The $B(G T)$ strength distribution from the IM-SRG interaction is shown in Fig. 11 (c). The IM-SRG interaction gives two strong peaks at energies $2.698 \mathrm{MeV}$ and $7.387 \mathrm{MeV}$. The IM-SRG interaction gives $\frac{3}{2}^{+}$g.s. for ${ }^{27} \mathrm{Si}$. Fig. 11 (d) shows the $B(G T)$ distribution from the USDB interaction. In this case we also get two strong peaks at $0.0 \mathrm{MeV}$ and $2.841 \mathrm{MeV}$ in ${ }^{27} \mathrm{Si}$. The USDB interaction gives $\frac{5}{2}^{+}$as the g.s. of ${ }^{27} \mathrm{Si}$, in agreement with the experiment. The comparison of accumulated sums of $B(G T)$ strengths for theoretical and the experimental values is shown in Fig. 11 (e). The USDB and IM-SRG interactions give same trend as the experimental data, while the CCEI method gives smaller values.

$$
\text { K. }{ }^{28} \mathbf{S i} \rightarrow{ }^{28} \mathbf{P}
$$

The experimental information on the distribution of $B(G T)$ strength is shown in Fig. 12. There are two experimental data sets available for the transition ${ }^{28} \mathrm{Si}$ $\rightarrow{ }^{28} \mathrm{P}$. The charge exchange reaction ${ }^{28} \mathrm{Si}\left({ }^{3} \mathrm{He}, t\right){ }^{28} \mathrm{P}$ was performed at $150 \mathrm{MeV} /$ nucleon, using the dispersionmatching technique [42] to get good energy resolution. In Fig. 12(a) the results for the ${ }^{28} \mathrm{Si}\left({ }^{3} \mathrm{He}, t\right){ }^{28} \mathrm{P}$ reaction are shown up to the excitation energy $5.57 \mathrm{MeV}$. The shell model study has already been carried out [42] by using Wildenthal's USD interaction. In this figure, a large $B(G T)$ strength is obtained at the excitation energy $2.15 \mathrm{MeV}$, but this value is normalized to the $(p, n)$ data which is taken from Ref. [34]. The $B(G T)$ distribution from the ${ }^{28} \mathrm{Si}(p, n){ }^{28} \mathrm{P}$ reaction performed at energy $136 \mathrm{MeV}$ per nucleon is given in Fig. 12(b). Here, a large $B(G T)$ strength is obtained at the excitation energy $2.10 \mathrm{MeV}$ of ${ }^{28} \mathrm{P}$. Fig. 12 (c) shows the theoretical results by using CCEI. We get a strong transition at 2.562 $\mathrm{MeV}$ with $B(G T)$ strength 0.82 , which comes from the transition ${ }^{28} \mathrm{Si}\left(0^{+}\right) \rightarrow{ }^{28} \mathrm{P}\left(1_{3}^{+}\right)$. We can also see many transitions above the excitation energy $4 \mathrm{MeV}$ of ${ }^{28} \mathrm{P}$, but they are very small in strength. The experimental g.s. of ${ }^{28} \mathrm{P}$ is $3^{+}$, while the CCEI predicts $0^{+}$. Fig. $12(\mathrm{~d})$ shows the $B(G T)$ strength distribution from the IM-SRG interaction. Here, we see a strong peak at the excitation energy $2.056 \mathrm{MeV}$ of ${ }^{28} \mathrm{P}$, which comes from the transition ${ }^{28} \mathrm{Si}\left(0^{+}\right) \rightarrow{ }^{28} \mathrm{P}\left(1_{3}^{+}\right)$. The IM-SRG interaction predicts $2^{+}$as the g.s. of ${ }^{28} \mathrm{P}$. In Fig. $12(\mathrm{e})$, the $B(G T)$ strength distribution obtained with the USDB interaction is shown. In this case, we can see two comparable peaks at excitation energies 2.065 and $4.847 \mathrm{MeV}$ of ${ }^{28} \mathrm{P}$, which come from the transition ${ }^{28} \mathrm{Si}\left(0^{+}\right) \rightarrow{ }^{28} \mathrm{P}\left(1_{3}^{+}\right)$and ${ }^{28} \mathrm{Si}\left(0^{+}\right) \rightarrow{ }^{28} \mathrm{P}\left(1_{7}^{+}\right)$, respectively. The USDB interaction gives $3^{+}$g.s. for ${ }^{28} \mathrm{P}$, in agreement with the experiment. In Fig. 12(f), the accumulated sums of $B(G T)$ strengths is shown. The USDB interaction shows a similar trend as the experimental data. The IM-SRG interaction gives 


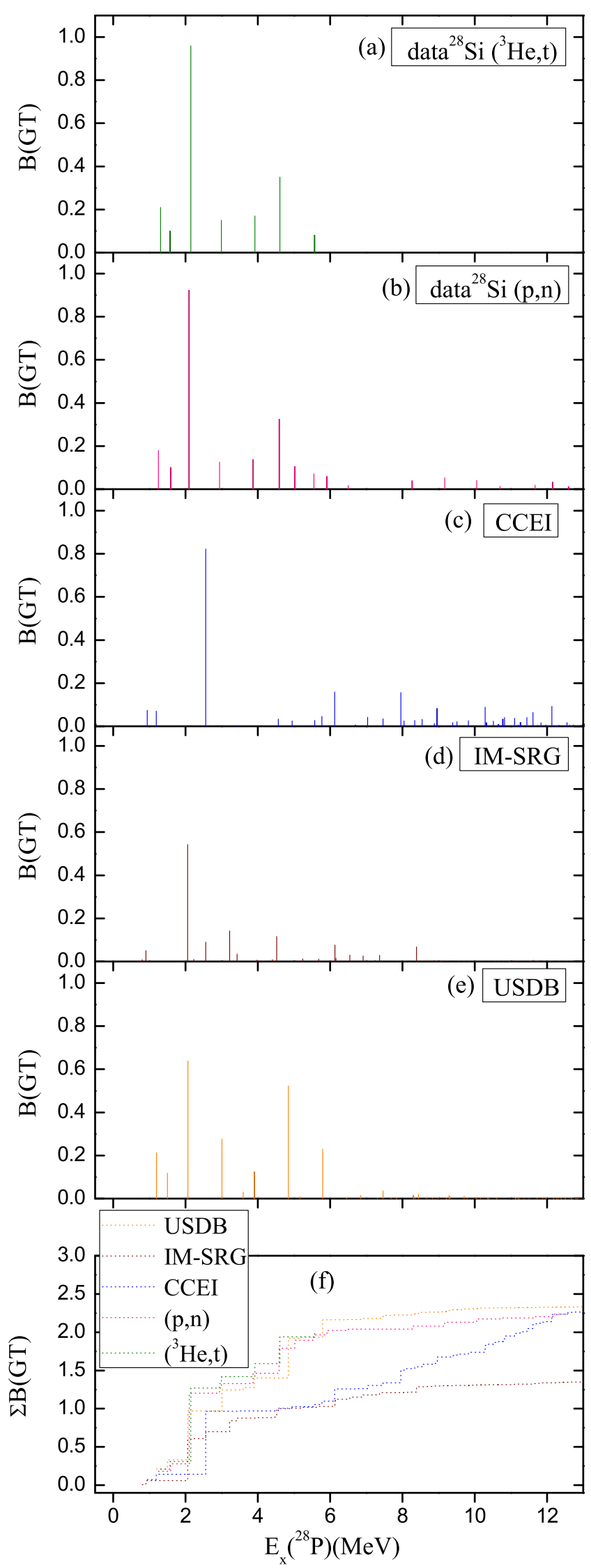

smaller value in comparison with the other interactions and the experimental data.

$$
\text { L. }{ }^{31} \mathbf{P} \rightarrow{ }^{31} \mathbf{S i}
$$

The $B(G T)$ strength distribution for the transition ${ }^{31} \mathrm{P}$ $\rightarrow{ }^{31} \mathrm{Si}$ is shown in Fig. 13. In Fig. 13(a), the experimental data is shown for the reaction ${ }^{31} \mathrm{P}(n, p){ }^{31} \mathrm{Si}$ [43]. In the experimental data, we see an intense peak at the excitation energy $5 \mathrm{MeV}$ of ${ }^{31} \mathrm{Si}$. The charge exchange reaction ${ }^{31} \mathrm{P}(n, p){ }^{31} \mathrm{Si}$ was performed to find the double differential cross section with the incident neutron energy of $198 \mathrm{MeV}$. Using multipole decomposition techniques the $B(G T)$ strength distribution was extracted. The shell model study using the universal $s d$ (USD) interaction has already been done in Ref. [43]. Fig. 13(b) shows the $B(G T)$ distribution obtained with the CCEI method. We see the strongest peak at the excitation energy $3.557 \mathrm{MeV}$ of ${ }^{31} \mathrm{Si}$, which comes from the transition ${ }^{31} \mathrm{P}\left(\frac{1}{2}_{1}^{+}\right) \rightarrow{ }^{31} \mathrm{Si}\left(\frac{3}{2}_{2}^{+}\right)$. The strength of this transition is very small in comparison to the strength of the strongest peak in the experiment. The CCEI gives $\frac{3}{2}^{+}$g.s. for ${ }^{31} \mathrm{Si}$, which agrees with the experiment. In Fig. 13(c), the distribution of the $B(G T)$ strength is shown for the IM-SRG interaction. Here, we see the strongest peak at the excitation energy $4.685 \mathrm{MeV}$ of ${ }^{31} \mathrm{Si}$, which comes from the transition ${ }^{31} \mathrm{P}\left(\frac{1}{2}_{1}^{+}\right) \rightarrow{ }^{31} \mathrm{Si}\left(\frac{1}{2}_{3}^{+}\right)$, but also here the strength is very small compared to the strongest peak in the experimental data. The IM-SRG also reproduces correctly the experimental g.s. of ${ }^{31} \mathrm{Si}$. Fig. 13(d) shows the $B(G T)$ distribution from the USDB interaction. In this case the strongest peak is observed at the excitation energy $4.661 \mathrm{MeV}$ with strength 0.30 , which is larger than the strength of the peaks obtained in the ab initio interactions. This peak comes from the transition ${ }^{31} \mathrm{P}\left(\frac{1}{2}_{1}^{+}\right) \rightarrow$ ${ }^{31} \operatorname{Si}\left(\frac{1}{2}_{2}^{+}\right)$. The USDB interaction also gives the correct g.s. of ${ }^{31} \mathrm{Si}$. Fig. $13(\mathrm{e})$ shows the accumulated $B(G T)$ strengths for all three interactions and the experimental data. All three interactions give small values in comparison to the experimental data.

$$
\text { M. }{ }^{32} \mathbf{S} \rightarrow{ }^{32} \mathbf{P}
$$

Fig. 14 presents the experimental and theoretical information on the distribution of the $B(G T)$ strength for the transition ${ }^{32} \mathrm{~S} \rightarrow{ }^{32} \mathrm{P}$. For the experimental data, the charge exchange reaction ${ }^{32} \mathrm{~S}\left(d,{ }^{2} \mathrm{He}\right){ }^{32} \mathrm{P}$ was performed at forward angles and at an incident energy of $E_{d}=170$ $\mathrm{MeV}$ with a resolution of $150 \mathrm{keV}$ [44]. Fig. 14 (a) shows the experimental data for $B(G T)$ strength distribution. Here, we see an intense peak at the excitation energy 4.2 $\mathrm{MeV}$ of ${ }^{32} \mathrm{P}$. The experimental g.s. of ${ }^{32} \mathrm{P}$ is $1^{+}$. Fig. 14 (b) presents the results obtained by using the CCEI. Here, we see three strong peaks at excitation energies $4.573 \mathrm{MeV}, 5.494 \mathrm{MeV}$ and $6.741 \mathrm{MeV}$ in ${ }^{32} \mathrm{P}$, which

FIG. 12: Comparison of the experimental and theoretical $B(G T)$ distributions for ${ }^{28} \mathrm{Si} \rightarrow{ }^{28} \mathrm{P}$. 
TABLE II: Comparison between the experimental and theoretical centroid energy of GT distributions for $s d$ shell nuclei.

\begin{tabular}{|c|c|c|c|c|c|c|c|c|c|c|c|}
\hline S.No. & Initial & Final & $\beta$-decay & $(n, p)$ & $\left(d,{ }^{2} \mathrm{He}\right)$ & $\left(t,{ }^{3} \mathrm{He}\right)$ & $\left({ }^{3} \mathrm{He}, t\right)$ & $(p, n)$ & CCEI & IM-SRG & USDB \\
\hline 1. & ${ }^{20} \mathrm{Ne}\left(0^{+}\right)$ & ${ }^{20} \mathrm{~F}\left(1^{+}\right)$ & & 5.39 & & & & & 4.66 & 4.92 & 5.08 \\
\hline 2. & ${ }^{23} \mathrm{Na}\left(\frac{3}{2}^{+}\right)$ & ${ }^{23} \mathrm{Mg}\left(\frac{1}{2}^{+}, \frac{3}{2}^{+}, \frac{5}{2}^{+}\right)$ & & & & & 5.00 & & 5.86 & 5.19 & 5.59 \\
\hline 3. & ${ }^{23} \mathrm{Na}\left(\frac{3}{2}^{+}\right)$ & ${ }^{23} \mathrm{Ne}\left(\frac{1}{2}^{+}, \frac{3}{2}^{+}, \frac{5}{2}^{+}\right)$ & & 3.01 & & & & & 1.63 & 1.81 & 2.10 \\
\hline 4. & ${ }^{24} \mathrm{Mg}\left(0^{+}\right)$ & ${ }^{24} \mathrm{Na}\left(1^{+}\right)$ & & & 2.73 & 3.22 & & & 1.96 & 1.67 & 2.82 \\
\hline 5. & ${ }^{24} \operatorname{Mg}\left(0^{+}\right)$ & ${ }^{24} \mathrm{Al}\left(1^{+}\right)$ & & & & & 2.44 & 2.21 & 2.35 & 1.50 & 2.28 \\
\hline 6. & ${ }^{25} \mathrm{Mg}\left(\frac{5}{2}^{+}\right)$ & ${ }^{25} \mathrm{Al}\left(\frac{3}{2}^{+}, \frac{5}{2}^{+}, \frac{7}{2}^{+}\right)$ & & & & & 1.93 & & 2.33 & 2.00 & 0.88 \\
\hline 7. & ${ }^{26} \operatorname{Mg}\left(0^{+}\right)$ & ${ }^{26} \mathrm{Na}\left(1^{+}\right)$ & & & 1.74 & 1.90 & & & 2.20 & 1.82 & 1.58 \\
\hline 8. & ${ }^{26} \operatorname{Mg}\left(0^{+}\right)$ & ${ }^{26} \mathrm{Al}\left(1^{+}\right)$ & & & & & 6.08 & 5.95 & 8.47 & 6.40 & 6.87 \\
\hline 9. & ${ }^{26} \mathrm{Si}\left(0^{+}\right)$ & ${ }^{26} \mathrm{Al}\left(1^{+}\right)$ & 1.44 & & & & & & 1.24 & 1.37 & 1.32 \\
\hline 10. & ${ }^{27} \mathrm{Al}\left(\frac{5}{2}^{+}\right)$ & ${ }^{27} \mathrm{Si}\left(\frac{3}{2}^{+}, \frac{5}{2}^{+}, \frac{7}{2}^{+}\right)$ & & & & & 5.35 & & 7.29 & 5.60 & 5.37 \\
\hline 11. & ${ }^{28} \operatorname{Si}\left(0^{+}\right)$ & ${ }^{28} \mathrm{P}\left(1^{+}\right)$ & & & & & 2.80 & 3.64 & 6.08 & 3.82 & 3.66 \\
\hline 12. & ${ }^{31} \mathrm{P}\left(\frac{1}{2}^{+}\right)$ & ${ }^{31} \mathrm{Si}\left(\frac{1}{2}^{+}, \frac{3}{2}^{+}\right)$ & & 3.53 & & & & & 2.61 & 4.19 & 3.39 \\
\hline 13. & ${ }^{32} \mathrm{~S}\left(0^{+}\right)$ & ${ }^{32} \mathrm{P}\left(1^{+}\right)$ & & & 4.10 & & & & 5.36 & 3.45 & 5.31 \\
\hline
\end{tabular}

come from the transitions ${ }^{32} \mathrm{~S}\left(0^{+}\right) \rightarrow{ }^{32} \mathrm{P}\left(1_{3}^{+}\right),{ }^{32} \mathrm{~S}\left(0^{+}\right)$ $\rightarrow{ }^{32} \mathrm{P}\left(1_{4}^{+}\right)$and ${ }^{32} \mathrm{~S}\left(0^{+}\right) \rightarrow{ }^{32} \mathrm{P}\left(1_{5}^{+}\right)$, respectively. The CCEI predicts the g.s. of ${ }^{32} \mathrm{P}$ as $2^{+}$, while the experimental g.s. is $1^{+}$. Fig. 14 (c) shows the $B(G T)$ distribution obtained by using the IM-SRG interaction. Here, we notice a peak at excitation energy $3.642 \mathrm{MeV}$ of ${ }^{32} \mathrm{P}$, which comes from the transition ${ }^{32} \mathrm{~S}\left(0^{+}\right) \rightarrow{ }^{32} \mathrm{P}\left(1_{5}^{+}\right)$, weak in comparison to the the strongest peak of the experimental data and the CCEI results. The IM-SRG interaction give $0^{+}$as the g.s. of ${ }^{32} \mathrm{P}$. Fig. 14 (d) shows the $B(G T)$ distribution obtained by using the phenomenological USDB interaction. This interaction gives two peaks with comparable strengths, but weak compared to the strongest peak from the experimental data. The strong peaks with the USDB interaction are at excitation energies 5.33 MeV and $6.019 \mathrm{MeV}$ of ${ }^{32} \mathrm{P}$, which come from the transitions ${ }^{32} \mathrm{~S}\left(0^{+}\right) \rightarrow{ }^{32} \mathrm{P}\left(1_{6}^{+}\right)$and ${ }^{32} \mathrm{~S}\left(0^{+}\right) \rightarrow{ }^{32} \mathrm{P}\left(1_{7}^{+}\right)$, respectively. The USDB interaction gives $3^{+}$as the g.s. of ${ }^{32} \mathrm{P}$. Fig. 14 (e) shows the accumulated sums of $B(G T)$ strength. Among the three interactions, the CCEI gives better results for the accumulated sums.

\section{N. Centroid energies}

In Sects. III.A-III.M, we discussed GT distributions for $s d$ shell nuclei obtained with the two ab initio interactions as well as the USDB. Among the three interactions, the USDB in general gives the best account of the experimental data. The $a b$ initio interactions give rather reasonable account of the experimental data, though there are deviations in many cases. In Table II, we show a comparison between the experimental and theoretical centroid energies of the GT distributions for $s d$ shell nuclei.

$A b$ initio interactions give larger GT strength than USDB and the experimental data in the lower excitation energy region, for example, in the ${ }^{23} \mathrm{Na} \rightarrow{ }^{23} \mathrm{Ne}$ and ${ }^{24} \mathrm{Mg} \rightarrow{ }^{24} \mathrm{Na}(<1 \mathrm{MeV})$ transitions. On the other hand, less GT strength is seen in lower excitation energy region, for example, in the ${ }^{25} \mathrm{Mg} \rightarrow{ }^{25} \mathrm{Al}$ and ${ }^{26} \mathrm{Mg} \rightarrow$ ${ }^{26} \mathrm{Na}$ transitions. This is also true for the ${ }^{26} \mathrm{Mg} \rightarrow{ }^{26} \mathrm{Al}$ and ${ }^{27} \mathrm{Al} \rightarrow{ }^{27} \mathrm{Si}$ transitions in case of CCEI. These differences are reflected in the centroid energies of the GT distributions. They are smaller (larger) when more (less) strength is found in the lower excitation energy region as shown in Table II. In case of the $a b$ initio interactions, single-particle energy gap between $d_{3 / 2}$ and $d_{5 / 2}$ orbits is large compared to USDB, in particular for CCEI. This could explain the general feature that ab initio interactions show much lower strength at low energies in higher mass nuclei. Especially small GT strengths for CCEI in the transitions shown in Figs. 9-11 can be attributed to insufficient contributions from $d_{3 / 2}$ orbit due to the largest gap among the interactions. As discussed in Sect. II, deviations of the GT strength, calculated by using the $a b$ initio interactions, from the experimental data become generally larger for the higher mass nuclei because of the lack of three-body cluster terms among valence nucleons.

In the present calculations, we used the one-body GT operator with a universal quenching factor for both ab initio and phenomenological USDB interactions. However, the GT operator should be evolved in the same way as the Hamiltonians for the IM-SRG and CCEI methods. This gives rise to induced two-body operators [58]. Although this effect is taken into account here by adopting a phenomenological universal quenching factor for the one-body operator, induced two-body operators can lead to mass dependent quenching factors, which may also depend on the interactions. The present calculation, therefore, has limitations because of the truncations of the Hamiltonians up to the two-body terms, and the operator up to the one-body term. 


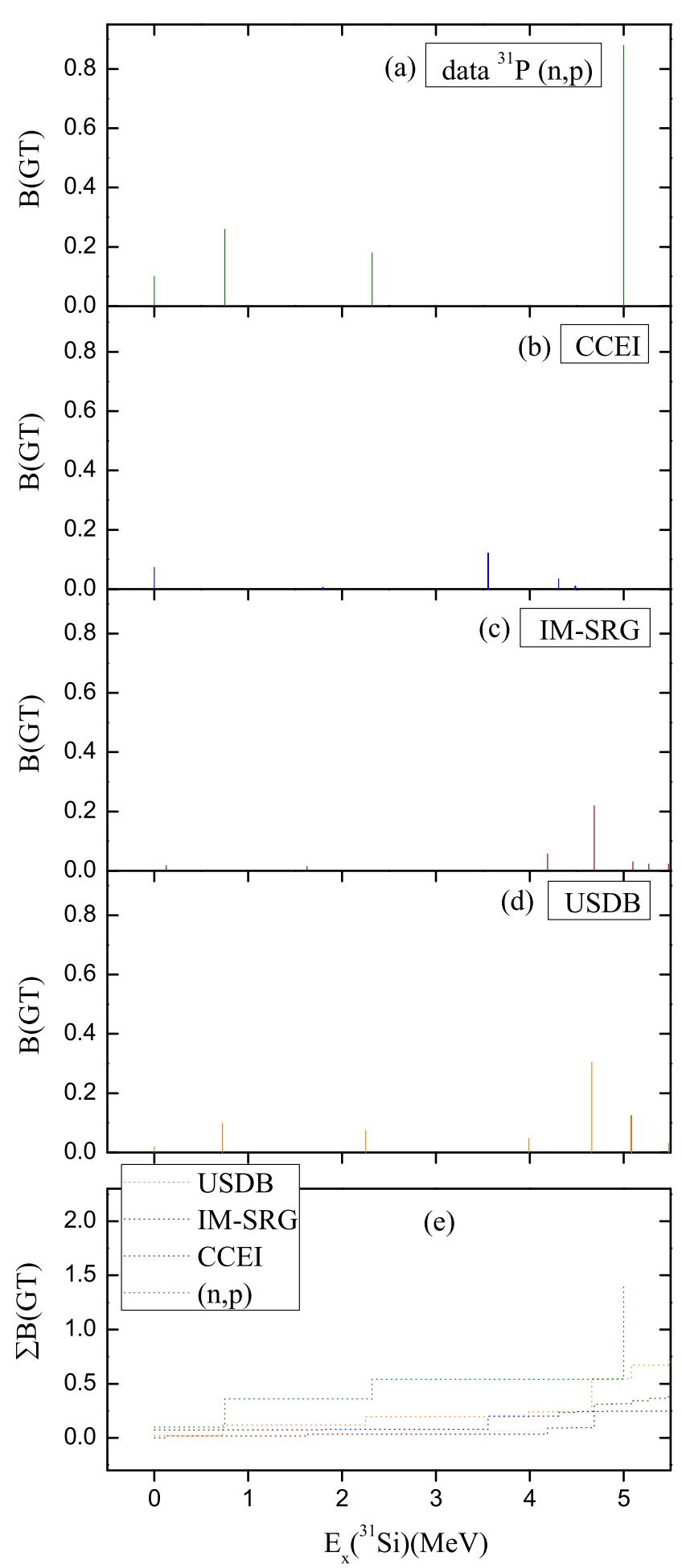

FIG. 13: Comparison of the experimental and theoretical $B(G T)$ distributions for ${ }^{31} \mathrm{P} \rightarrow{ }^{31} \mathrm{Si}$.

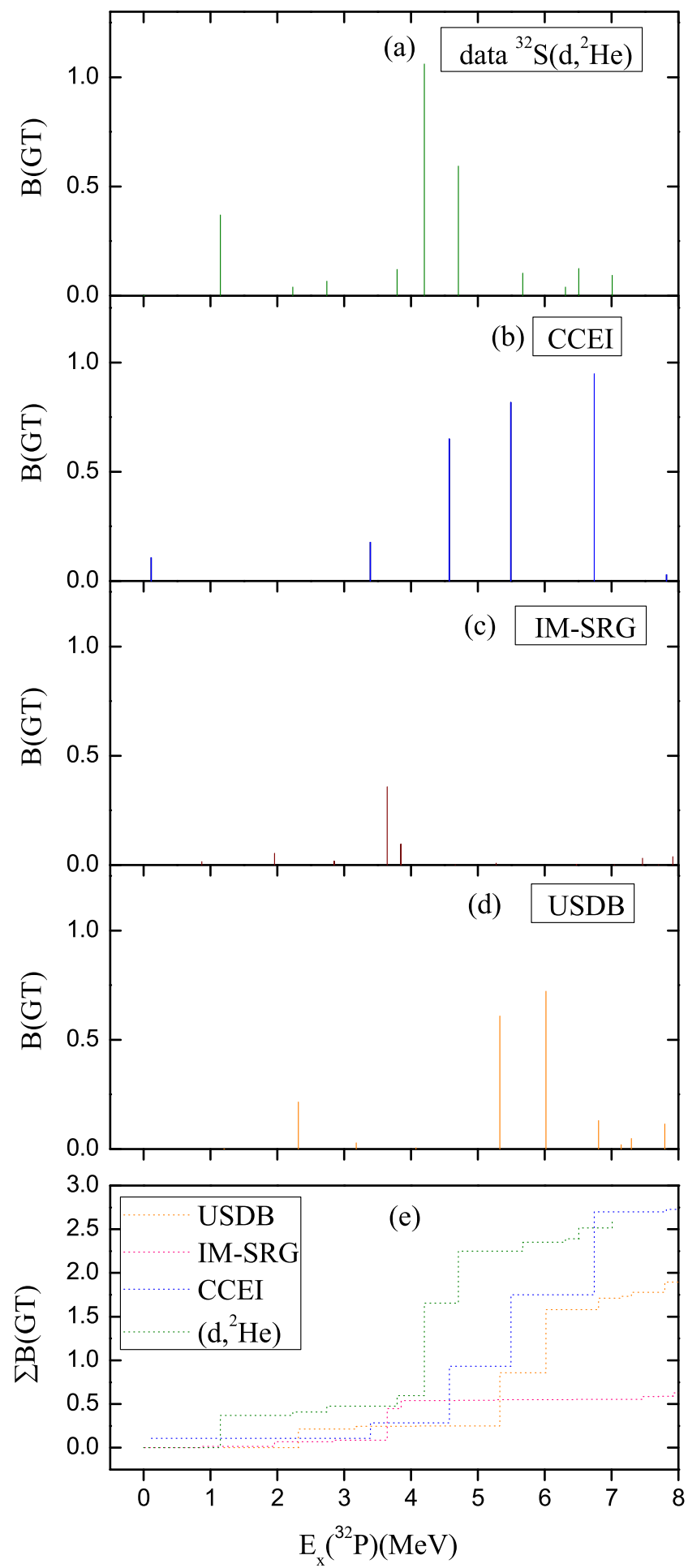

FIG. 14: Comparison of the experimental and theoretical $B(G T)$ distributions for ${ }^{32} \mathrm{~S} \rightarrow{ }^{32} \mathrm{P}$. 


\section{ELECTRON CAPTURE RATES IN ${ }^{23}$ NA AND ${ }^{25} \mathrm{MG}$}

In this section, we apply the GT transition strengths obtained by the ab initio effective interactions in $s d$ shell nuclei to evaluate the electron capture rates in stellar environments.

Electron capture rates at high densities and high temperatures are evaluated as [59-62]

$$
\begin{gathered}
\lambda=\frac{\ln 2}{6146(s)} \sum_{i} W_{i} \sum_{f} B(G T ; i \rightarrow f) \\
\times \int_{\omega_{\text {min }}}^{\infty} \omega p\left(Q_{i f}+\omega\right)^{2} F(Z, \omega) S_{e}(\omega) d \omega, \\
Q_{i f}=\left(M_{\mathrm{p}} c^{2}-M_{\mathrm{d}} c^{2}+E_{i}-E_{f}\right) / m_{e} c^{2}, \\
W_{i}=\left(2 J_{i}+1\right) e^{-E_{i} / k T} / \sum_{i}\left(2 J_{i}+1\right) e^{-E_{i} / k T},
\end{gathered}
$$

where $\omega$ and $p$ are electron energy and momentum in units of $m_{e} c^{2}$ and $m_{e} c ; M_{\mathrm{p}}$ and $M_{\mathrm{d}}$ are the masses of parent and daughter nuclei, and $E_{i}$ and $E_{f}$ are excitation energies of initial and final states. $F(Z, \omega)$ is the Fermi function, and $S_{e}(\omega)$ is the Fermi-Dirac distribution for electrons, where the chemical potential $\left(\mu_{e}\right)$ is determined from the density $\left(\rho Y_{e}\right)$ by

$$
\rho Y_{e}=\frac{1}{\pi^{2} N_{A}}\left(\frac{m_{e} c}{\hbar}\right)^{3} \int_{0}^{\infty}\left(S_{e}-S_{p}\right) p^{2} d p .
$$

Here $N_{A}$ is the Avogadro number, and $S_{p}$ is the FermiDirac distribution for positrons with the chemical potential $\mu_{p}=-\mu_{e}$. Its value can become as large as 2,5 and $11 \mathrm{MeV}$ at high densities $\rho Y_{e}=10^{8}, 10^{9}$ and $10^{10}$ $\mathrm{g} / \mathrm{cm}^{3}$, respectively, decreasing slightly as the temperature increases. The reaction rates become larger at higher densities because of the larger chemical potential.

Here, we evaluate the electron capture rates on ${ }^{23} \mathrm{Na}$ and ${ }^{25} \mathrm{Mg}$. These rates are important in the study of the nuclear URCA processes that determine the cooling of the O-Ne-Mg core of stars with initial masses of 8-10 $\mathrm{M}_{\odot}$ $[63,64]$. The electron-capture rates for ${ }^{23} \mathrm{Na}\left(\mathrm{e}^{-}, \nu\right)^{23} \mathrm{Ne}$ are evaluated by using the $B(G T)$ strengths obtained from the IM-SRG and CCEI methods for densities $\rho Y_{e}$ $=10^{8}, 10^{9}$ and $10^{10} \mathrm{~g} / \mathrm{cm}^{3}$ and temperatures $\mathrm{T}=10^{8.7}$. $10^{9.6}$ K. The GT transitions from $3 / 2^{+}$(g.s.) and $5 / 2^{+}(0.440 \mathrm{MeV})$ states in ${ }^{23} \mathrm{Na}$ are included. The calculated rates are shown in Fig. 15. Here the same quenching factor $f_{q}=0.77$ is used for all three interactions. The rates calculated by using the IM-SRG and CCEI methods are large compared with the USDB results. In the USDB* interaction shown in Fig. 15, the available experimental energies and $B(G T)$ strengths are taken into account, so the USDB* is more realistic [64]. Our results for the IM-SRG are close to those for the USDB*.

\section{${ }^{23} \mathrm{Na}\left(\mathrm{e}^{-}, \mathrm{v}\right){ }^{23} \mathrm{Ne}$}

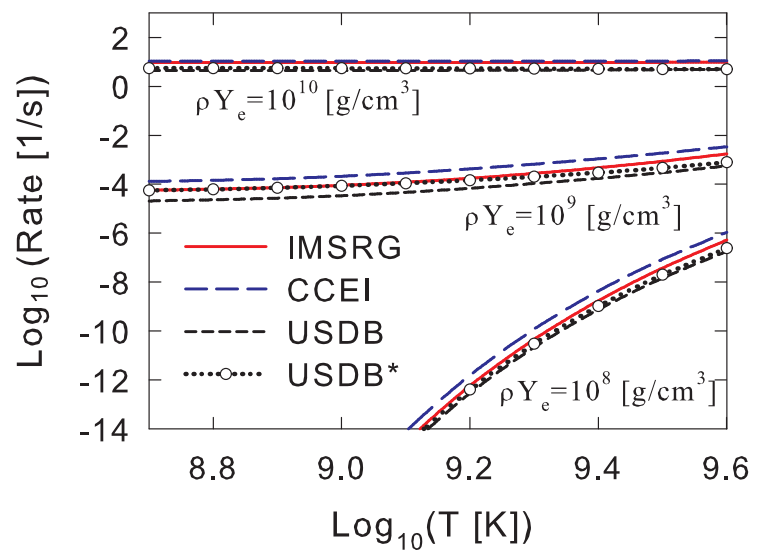

FIG. 15: Calculated electron capture rates on ${ }^{23} \mathrm{Na}$ obtained by shell model calculations with different effective interactions.

This comes from the fact that the $B(G T)$ value for the transition from the g.s. of ${ }^{23} \mathrm{Na}$ to the g.s. of ${ }^{23} \mathrm{Ne}$ is close to the experimental value in case of IM-SRG, while it is smaller (larger) in case of USDB (CCEI). Both IMSRG and CCEI give larger $B(G T)$ than USDB and the experiment for $E_{x}=0.5-3.5 \mathrm{MeV}$. Compared to USDB and USDB*, this leads to an enhancement of the capture rates by about a factor 2 at higher densities, $\rho Y_{e}=10^{10}$ $\mathrm{g} / \mathrm{cm}^{3}$. Since the dominant contribution to the capture rates for ${ }^{23} \mathrm{Na}\left(e^{-}, \nu\right){ }^{23} \mathrm{Ne}$ comes from the g.s. to g.s. transition [63, 64], IM-SRG is practically applicable to the evaluation of the weak rates in stellar environment, in spite of the enhanced $B(G T)$ strength at $E_{x}=0.5-3.5$ $\mathrm{MeV}$. The results calculated from the CCEI are enhanced compared to the USDB* by a factor of $2-4$.

Electron-capture rates for ${ }^{25} \mathrm{Mg}\left(\mathrm{e}^{-}, \nu\right)^{25} \mathrm{Na}$ are shown in Fig. 16. The GT transitions from $5 / 2^{+}$(g.s.), $1 / 2^{+}(0.588 \mathrm{MeV})$ and $3 / 2^{+}(0.975 \mathrm{MeV})$ states in ${ }^{25} \mathrm{Mg}$ are taken into account. The rates calculated with the CCEI and IM-SRG are close to those of the USDB* within a factor of 2 . We thus find that the GT strengths obtained by the ab initio interactions are reasonably valid for the evaluation of the weak rates at high densities and high temperatures for the lower mass $s d$ shell nuclei considered here. 


\section{${ }^{25} \mathrm{Mg}\left(\mathrm{e}^{-}, v\right){ }^{25} \mathrm{Na}$}

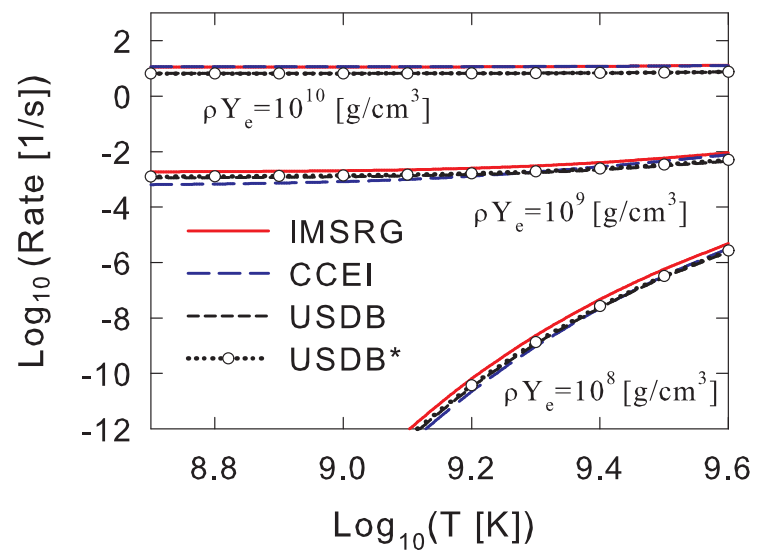

FIG. 16: Calculated electron capture rates on ${ }^{25} \mathrm{Mg}$ obtained by shell model calculations with different effective interactions.

\section{CONCLUSIONS}

In the present work we used $a b$ initio effective interactions to calculate the GT strengths in the $s d$ shell nuclei. The results of the USDB interaction show reason- able agreement with the available experimental data in comparison to ab initio effective interactions. Our work adds more information on the GT strength distributions obtained in earlier work. In some cases shifting of energy levels occurs, because $a b$ initio effective interactions are not able to reproduce correctly the excited states at the particular observed energies.

The GT calculated strengths are found to be applicable to evaluate nuclear weak rates for some lower mass $s d$ shell nuclei, such as ${ }^{23} \mathrm{Na}$ and ${ }^{25} \mathrm{Mg}$, within a factor of 2-4 in stellar environments. These nuclear weak rates play important roles in astrophysical processes. It is highly desirable to improve the ab initio method by including further the three-body valence cluster terms, that is, the terms IM-SRG(3) or $H_{3}^{A_{c}+3}$. It is also of interest to extend the method to include contributions from the twobody GT operators. In this work, they are taken into account by a phenomenological quenching factor for the one-body operator.

\section{Acknowledgments}

The authors would like to thank W. Bentz for the careful reading of the manuscript. We would like to thank P. Navrátil, S. R. Stroberg and G. R. Jansen for useful discussions on $a b$ initio effective interactions. AS acknowledges financial support from MHRD for her Ph.D. thesis work. PCS would like to thank financial support from faculty initiation grant. TS would like to thank a support from the Grants-in-Aid for Scientific Research (15K05090) of the MEXT of Japan.
[1] E. Caurier, A. Poves and A.P. Zuker, Missing and Quenched Gamow-Teller Strength, Phys. Rev. Lett. 74, 1517 (1995).

[2] A. L. Cole, T. S. Anderson, R. G. T. Zegers, Sam M. Austin, B. A. Brown, L. Valdez, S. Gupta, G. W. Hitt, and O. Fawwaz, Gamow-Teller strengths and electroncapture rates for $p f$-shell nuclei of relevance for late stellar evolution, Phys. Rev. C 86, 015809 (2012).

[3] P. Sarriguren, Contribution of excited states to stellar weak-interaction rates in odd- $A$ nuclei, Phys. Rev. C 93, 054309 (2016).

[4] A. Negret, T. Adachi, B. R. Barrett, C. Bumer, A. M. van den Berg, G. P. A. Berg, P. von Brentano, D. Frekers, D. De Frenne, H. Fujita et al., Gamow-Teller Strengths in the $A=14$ Multiplet: A Challenge to the Shell Model, Phys. Rev. Lett. 97, 062505 (2006).

[5] V. Kumar and P.C. Srivastava, Shell model description of Gamow-Teller strenghts in $p f$-shell nuclei, Eur. Phys. J. A 52, 181 (2016).

[6] V. Kumar, P.C. Srivastava and H. Li, Nuclear $\beta^{-}$decay half-lives for $f p$ and $f p g$ shell nuclei, J.Phys. G 43, 105104 (2016).

[7] K. Langanke and G. Martínez-Pinedo, Nuclear weakinteraction processes in stars, Rev. Mod. Phys. 75, 819
(2003)

[8] Y. Fujita, B. Rubio, and W. Gelletly, Spin-isospin excitations probed by strong, weak and electro-magnetic interactions, Prog. Part. Nucl. Phys. 66, 549 (2011), and references therein.

[9] K.G. Balasi, K. Langanke, G. Martínez-Pinedo, Neutrino-nucleus reactions and their role for supernova dynamics and nucleosynthesis, Prog. Part. Nucl. Phys. 85, 33 (2015).

[10] T. Suzuki, M. Honma, Hélène Mao, T. Otsuka and T. Kajino, Evaluation of electron capture reaction rates in $\mathrm{Ni}$ isotopes in stellar environments, Phys. Rev. C 83, 044619 (2011).

[11] G. Martínez-Pinedo, Y. H. Lam, K. Langanke, R. G. T. Zegers, and C. Sullivan, Astrophysical weak interaction rates for selected $A=20$ and $A=24$ nuclei, Phys. Rev. C 89, 045806 (2014).

[12] S. E. Woosley, D. H. Hartmann, R. D. Hoffman, and W. C. Haxton, The $\nu$-process, Astrophys. J. 356, 272 (1990).

[13] A. Heger, E. Kolbe, W. C. Haxton, K. Langanke, G. Martinez-Pinedo, and S. E. Woosley, Neutrino nucleosynthesis, Phys. Lett. B 606, 258 (2005).

[14] T. Suzuki and T. Kajino, Element synthesis in the supernova environment and neutrino oscillations, J. Phys. 
G 40, 083101 (2013).

[15] A. Byelikov, T. Adachi, H. Fujita, K. Fujita, Y. Fujita, K. Hatanaka, A. Heger, Y. Kalmykov, K. Kawase, K. Langanke et al., Gamow-Teller Strength in the Exotic Odd-Odd Nuclei ${ }^{138} \mathrm{La}$ and ${ }^{180} \mathrm{Ta}$ and Its Relevance for Neutrino Nucleosynthesis, Phys. Rev. Lett. 98, 082501 (2007).

[16] H. Hergert, S. K. Bogner, S. Binder, A. Calci, J. Langhammer, R. Roth, and A. Schwenk, In-medium similarity renormalization group with chiral two- plus three-nucleon interactions, Phys. Rev. C 87, 034307 (2013).

[17] D. J. Dean and M. Hjorth-Jensen, Coupled-cluster approach to nuclear physics, Phys. Rev. C 69, 054320 (2004).

[18] V. Soma, A. Cipollone, C. Barbieri, P. Navrátil, and T. Duguet, Chiral two- and three-nucleon forces along medium-mass isotope chains, Phys. Rev. C 89, 061301 (2014).

[19] S. R. Stroberg, H. Hergert, J. D. Holt, S. K. Bogner, and A. Schwenk, Ground and excited states of doubly openshell nuclei from ab initio valence-space Hamiltonians, Phys. Rev. C 93, 051301(R) (2016).

[20] P. Navrátil, V. G. Gueorguiev, J. P. Vary, W. E. Ormand, and A. Nogga, Structure of $A=10-13$ Nuclei with Two- Plus Three-Nucleon Interactions from Chiral Effective Field Theory, Phys. Rev. Lett. 99, 042501 (2007).

[21] P. Navrátil and W. E. Ormand, Ab initio shell model with a genuine three-nucleon force for the $p$-shell nuclei Phys. Rev. C 68, 034305 (2003).

[22] G. R. Jansen, M. D. Schuster, A. Signoracci, G. Hagen, and P. Navrátil, Open $s d$-shell nuclei from first principles, Phys. Rev. C 94, 011301(R) (2016).

[23] P.C. Srivastava and V. Kumar, Spectroscopic factor strengths using ab initio approaches, Phys. Rev. C 94, 064306 (2016).

[24] A. Saxena and P.C. Srivastava, First-principles results for electromagnetic properties of $s d$ shell nuclei, Phys. Rev. C 96, 024316 (2017).

[25] B. A. Brown and W. A. Richter, New USD Hamiltonians for the sd shell, Phys. Rev. C 74, 034315 (2006).

[26] B. A. Brown and W. D. M. Rae, The Shell-Model Code NuShellX@MSU, Nuclear Data Sheets 120, 115 (2014).

[27] S.K. Bonger, R.J. Frunstahl and A. Schwenk, From lowmomentum interactions to nuclear structure, Prog. Part. Nucl. Phys. 65, 94 (2010).

[28] K. Tsukiyama, S.K. Bogner and A. Schwenk, In-medium similarity renormalization group for nuclei, Phys. Rev. Lett. 106, 222502 (2011).

[29] B. W. Pointon, O. Häusser, R. Henderson, A. Celler, K. Hicks, K. P. Jackson, R. Jeppesen, B. Larson, J. Mildenberger, A. Trudel, et al., Gamow-Teller strength from the ${ }^{20} \mathrm{Ne}(n, p){ }^{20} \mathrm{~F}$ reaction at $E_{n}=198 \mathrm{MeV}$, Phys. Rev. C 44, 2430 (1991).

[30] Y. Fujita, Y. Shimbara, I. Hamamoto, T. Adachi, G. P. A. Berg, H. Fujimura, H. Fujita, J. Görres, K. Hara, K. Hatanaka et al., $M 1$ and Gamow-Teller transitions in $T=1 / 2$ nuclei ${ }^{23} \mathrm{Na}$ and ${ }^{23} \mathrm{Mg}$, Phys. Rev. C 66, 044313 (2002).

[31] B. Siebels, T. P. Gorringe, W. P. Alford, J. Bauer, J. Evans, S. El-Kateb, K. P. Jackson, A. Trudel, and S. Yen, Gamow-Teller strength in ${ }^{23} \mathrm{Na}(n, p)$ and a comparison to ${ }^{23} \mathrm{Na}\left(\mu^{-}, \nu\right)$, Phys. Rev. C 52, 1488 (1995).

[32] M. E. Howard, R. G. T. Zegers, Sam M. Austin, D. Bazin,
B. A. Brown, A. L. Cole, B. Davids, M. Famiano, Y. Fujita, A. Gade et al., Gamow-Teller strengths in ${ }^{24} \mathrm{Na}$ using the ${ }^{24} \mathrm{Mg}\left(t,{ }^{3} \mathrm{He}\right)$ reaction at $115 \mathrm{~A} \mathrm{MeV}$, Phys. Rev. C 78, 047302 (2008).

[33] S. Rakers, C. Bäumer, D. Frekers, R. Schmidt, A. M. van den Berg, V. M. Hannen, M. N. Harakeh, M. A. de Huu, H. J. Wörtche, D. De Frenne et al., Gamow-Teller matrix elements from the ${ }^{12} \mathrm{C}\left(d,{ }^{2} \mathrm{He}\right)$ and ${ }^{24} \mathrm{Mg}\left(d,{ }^{2} \mathrm{He}\right)$ reactions at $170 \mathrm{MeV}$, Phys. Rev. C 65, 044323 (2002).

[34] B. D. Anderson, N. Tamimi, A. R. Baldwin, M. Elaasar, R. Madey, D. M. Manley, M. Mostajabodda'vati, J. W. Watson, W. M. Zhang, and C. C. Foster, Gamow-Teller strength in the $(p, n)$ reaction at $136 \mathrm{MeV}$ on ${ }^{20} \mathrm{Ne},{ }^{24} \mathrm{Mg}$, and ${ }^{28} \mathrm{Si}$, Phys. Rev. C 43, 50 (1991).

[35] R. G. T. Zegers, R. Meharchand, T. Adachi, Sam M. Austin, B. A. Brown, Y. Fujita, M. Fujiwara, C. J. Guess, H. Hashimoto, K. Hatanaka et al., Spectroscopy of ${ }^{24} \mathrm{Al}$ and extraction of Gamow-Teller strengths with the ${ }^{24} \mathrm{Mg}\left({ }^{3} \mathrm{He}, t\right)$ reaction at $420 \mathrm{MeV}$, Phys. Rev. C 78, 014314 (2008).

[36] Y. Shimbara, Y. Fujita, T. Adachi, G. P. A. Berg, H. Fujita, K. Fujita, I. Hamamoto, K. Hatanaka, J. Kamiya, K. Nakanishi et al., Suppression of Gamow-Teller and $M 1$ transitions in deformed mirror nuclei ${ }^{25} \mathrm{Mg}$ and ${ }^{25} \mathrm{Al}$, Eur. Phys. J. A. 19, 25-31 (2004).

[37] R. G. T. Zegers, H. Akimune, Sam M. Austin, D. Bazin1, A. M.van den Berg, G. P. A. Berg, B. A. Brown, J. Brown, A. L. Cole, I. Daito et al., The $\left(t,{ }^{3} \mathrm{He}\right)$ and $\left({ }^{3} \mathrm{He}\right.$, $t)$ reactions as probes of Gamow-Teller strength, Phys. Rev. C 74, 024309 (2006).

[38] T. Niizeki, H. Ohnuma, T. Yamamoto, K. Katoh, T. Yamashita, Y. Hara, H. Okamura, H. Sakai, S. Ishida, N. Sakamoto et al., Spin-isospin excitation in $s d$-shell nuclei studied by the $\left(d,{ }^{2} \mathrm{He}\right)$ reaction at $E_{d}=270 \mathrm{MeV}, \mathrm{Nucl}$. Phys. A 577, 37 (1994).

[39] R. Madey, B. S. Flanders, B. D. Anderson, A. R. Baldwin, C. Lebo, J. W. Watson, Sam M. Austin, A. Galonsky, B. H. Wildenthal, and C. C. Foster, Gamow-Teller strength in the ${ }^{26} \mathrm{Mg}(p, n){ }^{26} \mathrm{Al}$ reaction at $135 \mathrm{MeV}$ and its fractionation into $T=0,1$, and 2 isospin channels, Phys. Rev. C 35, 2011 (1987); 36, 1647 (1987).

[40] Y. Fujita, Y. Shimbara, A. F. Lisetskiy, T. Adachi, G. P. A. Berg, P. von Brentano, H. Fujimura, H. Fujita, K. Hatanaka, J. Kamiya, et al., Analogous Gamow-Teller and $M 1$ transitions in ${ }^{26} \mathrm{Mg},{ }^{26} \mathrm{Al}$, and ${ }^{26} \mathrm{Si}$, Phys. Rev. C 67, 064312 (2003).

[41] Y. Fujita, H. Akimune, I. Daito, H. Fujimura, M. Fujiwara, M. N. Harakeh, T. Inomata, J. Jänecke, K. Katori, A. Tamii, et al., Mirror-symmetry structure of $A=27$, $T=1 / 2$ nuclei studied through strong, weak, and electromagnetic interactions, Phys. Rev. C 59, 90 (1999).

[42] Y. Fujita, H. Akimune, I. Daito, M. Fujiwara, M. N. Harakeh, T. Inomata, J. Jänecke, K. Katori, C. L?ttge, S. Nakayama et al., Isospin and spin-orbital structures of $J^{\pi}=1^{+}$states excited in ${ }^{28} \mathrm{Si}$, Phys. Rev. C 55, 1137 (1997).

[43] R. M. Sedlar, T. P. Gorringe, W. P. Alford, D. A. Beatty, J. Campbell, H. T. Fortune, P. Hui, D. A. Hutcheon, R. B. Ivie, K. P. Jackson et al., Gamow-Teller strength in $(n, p)$ charge exchange on ${ }^{31} \mathrm{P}$, Phys. Rev. C 59,789 (1999).

[44] E. W. Grewe, C. Bäumer, A. M. van den Berg, N. Blasi, B. Davids, D. De Frenne, D. Frekers, P. Haefner, M. N. Harakeh, M. Huynyadi, et al., Gamow-Teller transitions 
to ${ }^{32} \mathrm{P}$ studied through the ${ }^{32} \mathrm{~S}\left(d,{ }^{2} \mathrm{He}\right)$ reaction at $E_{d}=$ $170 \mathrm{MeV}$, Phys. Rev. C 69, 064325 (2004).

[45] D. R. Entem and R. Machleidt, Accurate chargedependent nucleon-nucleon potential at fourth order of chiral perturbation theory, Phys. Rev. C 68, 041001(R) (2003).

[46] D. R. Entem and R. Machleidt, Chiral effective field theory and nuclear forces, Phys. Rep. 503, 1 (2011).

[47] P. Navrátil, Local three-nucleon interaction from chiral effective field theory, Few-Body Syst. 41, 117 (2007).

[48] S. Okubo, Diagonalization of Hamiltonian and TammDancoff Equation, Prog. Theor. Phys. 12, 603 (1954).

[49] K. Suzuki, Construction of Hermitian Effective Interaction in Nuclei, Prog. Theor. Phys. 68, 246 (1982).

[50] P. Navrátil and B. R. Barret, No-core shell-model calculations with starting-energy-independent multivalued effective interactions, Phys. Rev. C 54, 2986 (1996).

[51] B.A. Brown and B.H. Wildenthal, Status of the Nuclear Shell Model, Annu. Rev. Nucl. Part. Sci. 38, 29 (1988).

[52] W. A. Richter, S. Mkhize, and B. A. Brown, sd-shell observables for the USDA and USDB Hamiltonians, Phys. Rev. C 78, 064302 (2008).

[53] B.A. Brown and B.H. Wildenthal, Corrections to the free-nucleon values of the single-particle matrix elements of the $M 1$ and Gamow-Teller operators, from a comparison of shell-model predictions with sd-shell data, Phys. Rev. C 28, 2397 (1983).

[54] B. H. Wildenthal, Empirical strengths of spin operators in nuclei, Prog. Part. Nucl. Phys. 11, 5 (1984).

[55] T. Suzuki, A. Arima, and K.-I. Kubo, Mixing of the $0 \mathrm{~g}$ orbit in ${ }^{20} \mathrm{Ne}$ and the analysis of a one-step forbidden transition, Nucl. Phys. A 288, 493 (1977).

[56] B. Jeckelmann, W. Beer, I. Beltrami, F.W.N. de Boer, G. de Chambrier, P.F.A. Goudsmit, J. Kern, H.J. Leisi, W. Ruckstuhl and A. Vacchi, Spectroscopic quadrupole moment of ${ }^{23} \mathrm{Na}$ from muonic X-rays, Nucl. Phys. A 408, 495 (1983); P. Raghavan, Table of nuclear moments, At.
Data Nucl. Data Tables 42, 189 (1989).

[57] K. Matsuta, T. Onishi, M. Fukuda, T. Minamisono, H. Akai, M. Sasaki, T. Yamaguchi, T.Miyake, K. Sato, K. Minamisono et al., Electromagnetic moments of short lived $\beta$ emitters ${ }^{21} \mathrm{~F},{ }^{23} \mathrm{Mg},{ }^{27} \mathrm{Si}$ and ${ }^{39} \mathrm{Ca}$, Hyperfine Interact. 120/121, 673 (1999).

[58] N. M. Parzuchowski, S. R. Stroberg, P. Navrátil, H. Hergert and S. K. Bogner, Ab initio electromagnetic observables with the in-medium similarity renormalization group, Phys. Rev. C 96, 034324 (2017).

[59] G. M. Fuller, W. A. Fowler, and M. J. Newton, Stellar weak-interaction rates for $s d$-shell nuclei. I - Nuclear matrix element systematics with application to Al-26 and selected nuclei of importance to the supernova problem, Astrophys. J. Suppl. Ser., 42, 447 (1980).

[60] G. M. Fuller, W. A. Fowler, and M. J. Newton, Stellar weak interaction rates for intermediate-mass nuclei. II $A=21$ to $A=60$, Astrophys. J. 252, 715 (1982).

[61] G. M. Fuller, W. A. Fowler, and M. J. Newton, Stellar weak interaction rates for intermediate mass nuclei. III Rate tables for the free nucleons and nuclei with $A=21$ to $A=60$, Astrophys. J. 48, 279 (1982).

[62] G. M. Fuller, W. A. Fowler, and M. J. Newton, Stellar weak interaction rates for intermediate-mass nuclei. IV Interpolation procedures for rapidly varying lepton capture rates using effective log $(f t)$-values, Astrophys. J. 293, 1 (1985).

[63] H. Toki, T. Suzuki, K. Nomoto, S. Jones, and R. Hirsci, Detailed $\beta$ transition rates for URCA nuclear pairs in 8-10 solar-mass stars, Physical Review C 88, 015806 (2013).

[64] T. Suzuki, H. Toki and K. Nomoto, Electron-capture and $\beta$-decay rates for $s d$-shell nuclei in stellar environments relevant to high-density O-Ne-Mg cores, Astrophys. J. 817, 163 (2016). 